\title{
Genomic and chemical decryption of the Bacteroidetes phylum for its potential to biosynthesize natural products
}

\section{Authors}

Stephan Brinkmann ${ }^{1}$, Michael Kurz², Maria A. Patras ${ }^{1}$, Christoph Hartwig1, Michael Marner $^{1}$, Benedikt Leis ${ }^{1}$, André Billion ${ }^{1}$, Yolanda Kleiner ${ }^{1}$, Armin Bauer ${ }^{2}$, Luigi Toti ${ }^{2}$, Christoph Pöverlein ${ }^{2}$, Peter E. Hammann ${ }^{3}$, Andreas Vilcinskas ${ }^{1,4}$, Jens Glaeser ${ }^{1,3}$, Marius S. Spohn $^{1 *}$ and Till F. Schäberle ${ }^{1,4^{*}}$

\section{Affiliations}

${ }^{1}$ Fraunhofer Institute for Molecular Biology and Applied Ecology (IME), Branch for Bioresources, 35392 Giessen, Germany

${ }^{2}$ Sanofi-Aventis Deutschland GmbH, 65926 Frankfurt am Main, Germany

${ }^{3}$ Evotec International GmbH, 37079 Göttingen, Germany

${ }^{4}$ Institute for Insect Biotechnology, Justus-Liebig-University Giessen, 35392 Giessen, Germany

* Correspondence: Marius S. Spohn marius.spohn@ime.fraunhofer.de; Till F. Schäberle till.f.schaeberle@agrar.uni-giessen.de

\begin{abstract}
With progress in genome sequencing and data sharing, 1000s of bacterial genomes are publicly available. Genome mining - using bioinformatics tools in terms of biosynthetic gene cluster (BGC) identification, analysis and rating - has become a key technology to explore the capabilities for natural product (NP) biosynthesis. Comprehensively, analyzing the genetic potential of the phylum Bacteroidetes revealed Chitinophaga as the most talented genus in terms of BGC abundance and diversity. Guided by the computational predictions, we conducted a metabolomics and bioactivity driven NP discovery program on 25 Chitinophaga strains. High numbers of peerless strain-specific metabolite buckets confirmed the upfront predicted biosynthetic potential and revealed a tremendous uncharted chemical space. Sourcing this dataset, we isolated the new iron chelating nonribosomally-synthesized cyclic tetradeca- and pentadecalipodepsipeptide antibiotics chitinopeptins with activity against Candida, produced by $C$. eiseniae DSM 22224 and $C$. flava KCTC 62435, respectively.
\end{abstract}

\section{Teaser}

Combination of omics-technologies revealed taxonomical hotspots for specialized metabolites within Bacteroidetes. 


\section{Introduction}

The steady utilization of antimicrobials in all areas affected by human and animal diseases but also in agriculture supports the distribution of resistance across all environmental niches(1-4). Consequently, mortality rates accumulate - caused by multi-resistant pathogens not treatable with approved anti-infectives(5). At the same time, approximately $15 \%$ of all crop production is lost to plant pathogen diseases nowadays $(6)$. With devastating socioeconomic consequences for human health and food supply security, the increase of antifungal drug resistances has become a global problem(7). To sustain the medical care and the food supply for a growing population, continued innovation in discovery of new lead anti-infectives with improved activities and/or novel mechanisms of action is of greatest need $(8,9)$. Small molecules from biological origin - natural products (NPs) - are a rich source of new chemical entities and have always been a major inspiration for the development of anti-infectives and control agents. Between 1981 and 2019,36.3\% of new medicines based on small molecules and approved by the US Food and Drug Administration (FDA) were NPs, derivatives of those or synthetic compounds that utilize an NP pharmacophore (10).

An underutilized as well as promising NP group is represented by the cyclic lipopeptides (CLPs), sharing a common structural core composed of a lipid tail linked to a cyclized oligopeptide(11). CLP biosynthesis is phylogenetically dispersed over the bacterial kingdom. This translates into an immense structural diversity arising from differences in amino acid sequences (length, type, configuration and modification) and the composition of the fatty acid moiety. Again, these variations result in heterogeneous biological activities of CLPs and promote their investigation in several research fields(11). For example recently isolated isopedopeptins(12) overcome multi-drug resistance of Gram-negative pathogens while daptomycin (13) $\left(\right.$ Cubicin $\left.^{\circledR}\right)$, an FDA-approved drug, is marketed for the treatment of complicated skin and soft-tissue infections caused by Gram-positive bacteria(14). Today, further promising CLPs are already under clinical investigation and attack multiple molecular targets(11). In contrast, other CLPs like fengycin(15) exhibit an intrinsic antifungal activity $(16,17)$ and several CLP-producing bacteria have been registered with the United States Environmental Protection Agency (EPA) for their application as biocontrol agents(18). Their biosynthesis and production is mostly investigated in the genera Pseudomonas, Bacillus, and Streptomyces $\operatorname{spp}(19)$. These taxa belong to the beststudied NP producers. Traditionally, antimicrobial NPs from bacterial origin had been isolated within large cultivation campaigns on those talented microorganisms. While those genera have delivered a plethora of structurally different NPs over the last decades, they represent only a limited phylogenetic diversity(20). In addition to classical approaches, computational evaluation of genomic data paved the way to discover novel NPs in a target oriented manner by applying bioinformatics tools for biosynthetic gene cluster (BGC) identification, analyzation and rating (21-26). These approaches provided evidence of a yet not fully exploited biosynthetic potential in terms of chemical novelty $(27-31)$ certified with complementary metabolomics studies $(32,33)$. The predicted level of novelty increases by shifting from classical NP producing taxa towards a to date yet underexplored phylogene tic and chemical space $(34,35)$. The validity of this dogma in NPdiscovery was recently shown, e.g. by the discovery of teixobactin(36) and darobactin(37), both produced by rare Proteobacteria.

The Bacteroidetes phylum represents such an underexplored phylogenetic space, too(34). Although, easy to cultivate and widely spread through all environments $(38,39)$ there are only a few biologically active molecules described from this taxonomic clade (e.g. isopedopeptins(12), elansolids(40), pinensins(41), formadicins(42), TAN-1057A-D(43), katanosins(44), and ariakemicins(45)). Whereas environmental studies already allowed a 
first glance at the genetic potential of the phylum(46), there is no systematic investigation of their actual genetic and metabolic repertoire and the overall number of isolated compounds remains low.

Motivated by this gap, we examined the NP biosynthesis potential of the Bacteroidetes phylum by computational analysis of 600 publically available genomes for their BGC amount, type, and diversity. This revealed the accumulation of NP production capability in only a few taxonomic hotspots rather than a general ability of the phylum members to produce complex secondary metabolites. The vast majority of Bacteroidetes BGC of the RiPPs, NRPS, PKS, and hybrid NRPS/PKS classes are non-connatural to any other phylum, in turn providing strong evidence of an overall high potential to discover novel scaffolds from this phylum. Particularly, the genus Chitinophaga represents an outstanding group, on average encoding 15.7 BGCs per strain and enriched in NRPS and PKS BGCs. Based on this analysis, we selected 25 members of this genus for a cultivation and screening program. Our systematic chemotype-barcoding matrix pointed towards a tremendous chemical space of new NPs within this dataset. Almost no known NPs were predicted, strengthening the upfront-performed computational strain evaluation and selection. Eventually, this process led to the discovery of a new class of nonribosomally-synthesized cyclic tetradeca- and pentadecalipodepsipeptides with iron chelating properties and candicidal activity. Those CLPs named chitinopeptins $\mathrm{A}, \mathrm{B}, \mathrm{C} 1+\mathrm{C} 2$, and $\mathrm{D} 1+\mathrm{D} 2$ were isolated from $C$. eiseniae DSM 22224 and $C$. flava KCTC 62435. The structures as well as the absolute stereochemistry of all amino acids were elucidated by extensive NMR studies and advanced Marfey's Analys is.

\section{Results}

\section{Bioinformatics analysis of the phylum Bacteroidetes}

The large and diverse phylum Bacteroidetes harbors Gram-stain-negative, chemoorganotrophic, non-spore forming rod shaped bacteria, graded into six classes. Members have colonized all types of habitats, including soil, ocean, freshwater, and the gastrointestinal tract of animals(38). Species from the mostly anaerobic Bacteroidia class are predominantly found in gastrointestinal tracts, while environmental Bacteroidetes belong primarily to the Flavobacteriia, Cytophagia, Chitinophagia, Saprospiria, and Sphingobacteriia classes $(47,48)$. Environmental studies based on amplicon diversity of adenylation and ketosynthase domains gave a first glance to the genetic potential of the phylum for the biosynthes is of complex NPs(46). In order to map the Bacteroidetes phylum systematically in terms of their BGC potential, we selected almost all publically available, closed, and correctly annotated genomes in addition with some whole genome shotgun (WGS) projects at the time of data processing, depicting all six classes. In total, 600 genomes were analyzed using the 'antibiotics and secondary metabolite analysis shell' (antiSMASH 5.0)(49). A phylogenetic tree based on complete $16 S$ ribosomal RNA (rRNA) genes was constructed to provide taxonomic context to the BGC comparisons (Fig. 1A). A multi value bar chart was placed around the tree displaying the total BGC amount of each strain, in parallel highlighting the specific amount of NRPS, PKS and hybrid BGCs of both types. Assigning their BGC amount and types over the phylogenetic tree enabled classes and genera comparison in terms of BGC amount. In most cases, a small linear positive correlation between genome size and number of secondary metabolite BGCs per genome is given, a phenomenon known from other bacteria(50) (Fig. 1A, B).

Mainly bacteria of the classes Bacteroidia and Flavobacteriia, with the smallest average genome size (3.78 and 3.51 Mbps) and an average BGC amount per strain (1.15 and 3.19 BGCs), display less significance for NP discovery. Exceptions are found in the genera Kordia (5.33 Mbps and 10 BGCs on average, three unique genomes analyzed $(\mathrm{n}=3)$ ) and 
Chryseobacteria (4.43 Mbps and 6.19 BGCs on average, $\mathrm{n}=48$ ) with up to five BGCs of the NRPS and/or PKS type. Many strains of these classes are pathogens and inhabit environments that are characterized by higher stability and lower complexity (e.g. guts)(38). NP production is an adaptive mechanism providing evolutionary fitness upon changing environmental conditions and in presence of growth competitors(51). In accordance, the most talented bacterial NP producers, like the Actinomycetes(30) and Myxobacteria(52) are mainly found in highly competitive environments as e.g. soils. This correlation can also be seen within the Bacteriodetes phylum. In contrast to the anaerobic and pathogenic species, a higher BGC load was observed in the freely living and aerobic classes. The Sphingobacteriia and Cytophagia classes have an average genome size of $5.55 \mathrm{Mbps}$ and $5.57 \mathrm{Mbps}$ respectively, and an average BGC load of 5.79 and 4.63 per strain. A remarkable outlier is the genus Pedobacter with up to 22 BGCs on a single genome. Nevertheless, our analysis revealed that the Chitinophagia class outcompetes the other phyla members in respect to BGC amount per genome. Summarized, the class matched up with 11.4 BGCs per strain and genomes of an average size of $6.64 \mathrm{Mbps}$. Within this class, the genus Chitinophaga $(\mathrm{n}=47)$ accumulates a markedly enriched amount of 15.7 BGCs per strain on an average genome size of $7.51 \mathrm{Mbps}$. $30 \%$ of their BGCs do belong to the classes of NRPS and PKS, including rare trans-AT PKS BGCs. The second best genus of the Bacteroidetes phylum in respect to BGC load is Taibaiella that also belongs to the Chitinophagia class, with a $27 \%$ smaller genome size and on average $19 \%$ less BGCs (Fig. 1C, D).

In order to discover novel chemistry, the pure amount of BGCs is of subordinate importance in comparison to the BGCs divergence, predicted to translate into structural diversity within the encoded metabolites $(20,53)$. Thus, we expanded the computational analysis by examining the sequential and compositional similarity of the BGCs detected in the 600 genomes using the 'biosynthetic gene similarity clustering and prospecting engine' (BiGSCAPE v1.0.0)(26). BiG-SCAPE creates a distance matrix by calculating the distance between every pair of BGC in the dataset. The distance matrix combines three metrics, the percentage of shared domain types (Jaccard index), the similarity between aligned domain sequences (Domain sequence similarity) and the similarity of domain pair types (Adjacency index). The comparative analysis of the Bacteroidetes BGCs with the integrated MIBiG (Minimum Information about a Biosynthetic Gene cluster, v1.4) database(54) enabled their correlation to 1,796-deposited BGCs and consequently the correlation of their synthesized metabolites. In total, in 415 of the 600 genomes analyzed, 2594 BGCs were detected and grouped with a default similarity score cutoff of $\mathrm{c}=0.6$ into a sequence similarity network with 306 gene cluster families (GCFs). A remarkable low number of only 12 GCFs clustered with known BGCs. Together, those 12 GCFs comprise $11.5 \%$ (298 BGCs) of all detected Bacteroidetes BGCs. Nine of them belonged to the BiG-SCAPE BGC classes of other PKS ("PKSother"), "Terpenes" or "Other". These GCFs encode known NP classes like biotin, ectoine, $\mathrm{N}$-acyl glycin, and eicosapentaenoic acid, as well as products of the NRPSindependent siderophore (NIS) synthetase type, precisely desferrioxamine and bisucaberin $\mathrm{B}(55)$, forming two connected though distinct clouds (Fig. 2). A Cytophagales specific GCF of the terpene class includes the MIBiG BGC0000650, encoding the carotenoid flexixanthin(56). The Bacteroidetes are well known producer of flexirubin-like pigments, which is reflected in a conserved biosynthesis across several classes $(57,58)$. The flexirubin gene cluster cloud (GCC) covers 268 BGCs from 252 individual strains and five of six analyzed classes. In our analysis, only the newly formed Saprospiria class(48) was an exception. However, considering that the analysis included only two Saprospiria strains it does not yet allow any integral assessment of its capabilities to produce these yellow pigments. The flexirubin GCC can be divided into at least five distinct GCFs. Resolved on 
class level this revealed a specific Flavobacteriia family including BGC0000838 from Flavobacterium johnsoniae UW101(57) as well as a specific Chitinophagia family including BGC0000839 from Chitinophaga pinensis DSM 2588(58). The latter in turn being directly connected to a third GCF, in majority covering BGCs from its genus Chitinophaga while not including a reference BGC.

In addition, the reference BGCs described to encode the bioactive NPs monobactam SQ 28, $332(59,60)$, elansolids $(40,61)$, and pinensins $(41)$, respectively, are annotated to three distinct GCFs (Fig. 2A). The monobactam BGC (BGC0001672) was unique and only identified in its described producer strain Flexibacter sp. ATCC 35103. Elansolids and pinensins represent patent protected chemical entities active against Gram-positive bacteria and filamentous fungi and yeasts, respectively. The complete elansolid encoding BGC (BGC0000178), almost $80 \mathrm{kbp}$ in size, was identified in the genomes of strain Chitinophaga sp. YR627 and Chitinophaga pinensis DSM 2588 (=DSM 28390(62)) with the genetic potential to produce elansolid already proposed for $C$.pinensis(63) (fig. S1A). Considering the restricted access to the original producer strain, Chitinophaga sancti DSM 21134(64), these strains provide alternative bioresources to access these polyketide-derived macrolides. In addition, both strains also harbor the pinensin BGC remarkably directly co-localized with the elansolid-type BGC. This co-localization leads to an artificial connectivity between both GCFs by using the chosen BiG-SCAPE parameters. Manual curation revealed in total six strains carrying a pinensin-like BGC in their genome (fig. S1B). The alignment of the RiPP core peptide revealed that only the amino acid sequence from strain Chitinophaga sp. YR627 was identical to the described pinensin sequence. The other strains show amino acid sequence variations, pointing towards structural variance (fig. S1C).

With > 200 GCFs identified and only 12 of them annotated towards known BGCs and their metabolites, the sequential and compositional similarity analysis revealed a tremendous BGC diversity and uniqueness within the Bacteroidetes phylum, remarkably differing from the composition of known BGCs deposited in the MiBIG database.

Expanding this similarity network analysis towards taxonomic relations on phylum level showed that there is no connaturality of Bacteroidetes BGC of the RiPPs, NRPS, PKS, and hybrid NRPS/PKS classes to any other phylum (Fig. 2B). This in turn provides a strong evidence of a general high potential to discover novel metabolites from this phylum. The majority ( $66 \%)$ of all GCFs belonging to the above mentioned BGC classes are found in the Chitinophagia class, only representing $13.7 \%$ of the analyzed strains. Within this class, the genus Chitinophaga can be prioritized in terms of BGC amount and composition. In respect of many more complete unique and novel RiPP, NRPS, PKS, and hybrid BGCs thereof (not depicted in the network), this is a strong indication that the biosynthetic potential within this genus is far from being fully exploited. It can be considered as the most promising starting point for the discovery of novel metabolites within this phylum.

\section{Metabolomics of the Chitinophaga}

Based on the genomic data evaluation we selected the Chitinophaga for performing a bioactivity guided NP discovery program. NPs are considered to be nonessential metabolites for bacterial growth and reproduction but rather providing evolutionary fitness, thus, being expressed as adaptive response to changing environmental conditions. Consequently, the discovered BGC potential is not expected to translate into the actually produced metabolite pattern under laboratory conditions(22). A common theme of strategies to approach this challenging incomplete link is the cultivation in several media variants exposing the strains to various stress conditions, like e.g. nutrient depletion $(65,66)$. As a consequence of nutrient depletion bacteria enter the stationary phase and reduce or even cease growth, often found to coincide with induction of secondary metabolite production(67). To trigger these events 
we cultivated a diversity of 25 Chitinophaga strains (tab. S1) in five different media for four as well as seven days.

The metabolites were extracted from freeze-dried culture broths with methanol and the organic extracts were subsequently analyzed by UHPLC-QTOF-UHR-MS. LC-MS datasets from a total of 250 extracts (and media controls) were examined allowing the definition of strain-specific molecular features. In an initial step, features (represented by $\mathrm{m} / \mathrm{z}$, retention time, isotope pattern) were calculated within all extracts. Curation of all datasets was necessary to filter background noise and confirm the authenticity of defined features. This curation step helped to avoid false uniqueness due to concentrations near the corresponding detection limit and to reduce the possibility of picking up background noise. Furthermore, the possibility of multiple mass spectrometric features for any NP contributes to the complexity of those datasets, e.g. by the formation of different ion adducts and in-sourcegenerated fragment ions of single molecules. The final dataset consisted of 93,526 features. Those were aligned into 4,188 buckets with a bucket being defined as an $\mathrm{m} / \mathrm{z}$ and retention time (RT) region hosting all features with matching $\mathrm{m} / \mathrm{z}$ and $\mathrm{RT}(68)$. We created a chemotype-barcoding matrix of this complex dataset, allowing its visualization and evaluation (Fig. 3A). After normalization of the dataset by buckets congruent with the media controls (in total 1,452), we determined in total 2,736 buckets as representing produced metabolites of the investigated Chitinophaga set.

The detected buckets were analyzed for presence in all combinations considering utilized cultivation media and incubation time. They were put in order according to $16 S \mathrm{rRNA}$ sequence similarity on strain level. In order to facilitate data interpretation and to identify strain specific as well as conserved metabolites all buckets were sorted according to their frequency of appearance. Comparative visualization of the short (four days) and prolonged (seven days) incubation time revealed differences on the chemotype profile of the strains. Most strains metabolize the majority of media components within the first four days of cultivation, recognized by only a small number of "media buckets" still present in the extracts after this incubation period. A second population of rather slow growing strains appeared to shift the metabolic profile only after seven days of incubation in comparison to the respective media control (e.g. C. caeni KCTC 62265, C. dinghuensis DSM 29821, C. niastensis DSM 24859, C. barathri, and C. cymbidii). Especially Chitinophaga sp. DSM 18078 required a prolonged incubation time to metabolize the media ingredients, producing $77.4 \%$ more metabolite buckets after seven days of incubation in comparison to the earlier sampling time. Within the whole dataset, on average 26.6\% (92.4) more metabolite buckets were detected after seven then after four days of incubation. In contrast, a fraction of bacterial metabolite buckets disappeared within the extracts of five strains after prolonged incubation, showing the necessity to vary cultivation conditions to access a possibly comprehensive metabolite profile of each investigated strain. The many media-specific buckets (colored) compared to the ones being produced in various media (black) also show the effect of variations of the bacterial nutrient supply (Fig. 3A). In combination with the varied cultivation period, this led to an average of approximately 46 unique buckets per strain. Especially C. flava KCTC 62435 and its close relative C. eiseniae DSM 22224 outcompeted the others by producing 305 and 150 unique metabolite buckets respectively (Fig. 3B), indicating their status as best-in-class producers. In total 1,154 buckets $(\sim 42 \%)$ of the entire dataset were identified only in one respective strain dataset, representing strainspecific and therefore unique metabolites (Fig. 3C). This high level of strain-specific metabolites shows the heterogeneity of the set used. In parallel, this experiment intrinsically depicts a structural diversity in terms of molecular size up to $5145.051 \mathrm{Da}$ and complete covered polarity range approachable by this procedure (fig. S2). 
Analysis of the conserved metabolites revealed 357 buckets to be present in at least 10 out of 25 strains while only the remarkably low number of seven buckets could be detected within samples of all Chitinophaga strains investigated. Based on their $\mathrm{MS}^{2}$-fragmentation patterns sharing the loss of long carbon chains, we postulated a structural relationship between four of them and assigned them as hitherto unknown (amino/phospho)-lipids.

Next, the complete LC-MS dataset was examined for the presence of structurally characterized microbial NPs $(\sim 1,700)$ deposited in our in-house database on the basis of accurate $m / z, \mathrm{RT}$, and isotope pattern. Strikingly, the frequency of rediscovery was zero. Considering the known bias of the database for natural products from classical NP producing taxa such as Actinobacteria, Myxobacteria, and fungi, this confirmed the very low congruency towards these taxa, which was also found by our BGC categorization study. A complementary scan of LC-MS/MS data of the entire dataset was compared with in silico fragments of $>40 \mathrm{k}$ NPs deposited in the commercial database AntiBase(69). Congruently, no database recorded NP was identified within our dataset besides the falcitidin(70), an acyltetrapeptide produced by a Chitinophaga strain. Although this finding shows the general applicability of this workflow, the underrepresentation of NPs isolated from the phylum Bacteriodetes also in public databases is still a severe limitation for comprehensive categorization of their omics-data today.

However, as a consequence, these data provided a high confidence level in the investigated strain portfolio and showed that the metabolite spectrum produced by these strains is largely underexplored and differs completely from the metabolites produced by classical NP producer taxa. Even though strains of the genus Chitinophaga are phylogenetically closely related, they produce an immense heterogeneity of metabolites, thereby, showing a high number of strain-specific metabolites, associated with a high likelihood for chemical novelty.

\section{Chitinopeptins, a new class of CLPs from C. eiseniae and C. flava}

A correlation of those untapped buckets with antibacterial activity was performed by screening the organic crude extracts against a panel of opportunistic microbial pathogens. In particular, methanol extracts of Chitinophaga eiseniae DSM 22224 and Chitinophaga flava KCTC 62435, the two strains with the highest level of metabolic uniqueness exhibited strong activity against Candida albicans FH2173. Bioactivity assay and UHPLC-UHR-ESI-MS guided fractionation led to the identification of six novel cyclic lipodepsipeptides. C. eiseniae produced the two tetradecalipodepsipeptides chitinopeptins $\mathrm{A}$ and $\mathrm{B}\left(\mathbf{1}\right.$ and 2) with molecular formulae $\mathrm{C}_{82} \mathrm{H}_{137} \mathrm{~N}_{17} \mathrm{O}_{28}\left(\mathbf{1},[\mathrm{M}+\mathrm{H}]^{+} 1809.0052\right)$ and $\mathrm{C}_{81} \mathrm{H}_{135} \mathrm{~N}_{17} \mathrm{O}_{28} \quad\left(2, \quad[\mathrm{M}+\mathrm{H}]^{+}\right.$1794.9907). Whereas, $C$. flava assembled the four pentadecalipodepsipeptides chitinopeptins $\mathrm{C} 1+\mathrm{C} 2$ and $\mathrm{D} 1+\mathrm{D} 2$ (3-6) with molecular formulae $\mathrm{C}_{84} \mathrm{H}_{140} \mathrm{~N}_{18} \mathrm{O}_{30}\left(\mathbf{3}\right.$ and 4, $[\mathrm{M}+\mathrm{H}]^{+}$1882.0177) and $\mathrm{C}_{83} \mathrm{H}_{138} \mathrm{~N}_{18} \mathrm{O}_{30}\left(\mathbf{5}\right.$ and $\mathbf{6},[\mathrm{M}+\mathrm{H}]^{+}$ 1868.0059) (Fig. 4). All compounds were present in the MS spectra as pairs of $[\mathrm{M}+3 \mathrm{H}]^{3+}$ and $[\mathrm{M}+2 \mathrm{H}]^{2+}$ ions (fig. S3A). All six native peptides appeared to be highly stable, since only poor yields of fragment ions arose using electrospray ionization source (fig. S3B), even under elevated collision energy conditions. Thereby, preventing MS/MS based structure prediction and structural relationship analysis using molecular networking.

The chemical structures of the six compounds were determined by extensive NMR studies using 1D- ${ }^{1} \mathrm{H}, 1 \mathrm{D}-{ }^{13} \mathrm{C}$, DQF-COSY, TOCSY, ROESY, multiplicity edited-HSQC, and HMBC spectra (Fig. 4). The analysis of the compounds in most "standard" NMR solvents was hampered by either extreme line broadening (DMSO, $\mathrm{MeOH}$, pyridine) or poor solubility $\left(\mathrm{H}_{2} \mathrm{O}\right.$, acetone). However, a mixture of $\mathrm{H}_{2} \mathrm{O}$ and $\mathrm{CD}_{3} \mathrm{CN}$ (ratio 1:1) gave rise to NMR spectra of high quality and confirmed the presence of peptides. In order to obtain a good dispersion of the amide resonances ${ }^{1} \mathrm{H}$-spectra were acquired at different temperatures 
between 290 and $305 \mathrm{~K}$. A temperature of $299 \mathrm{~K}$ or $300 \mathrm{~K}$, respectively, was found to be the best compromise considering signal dispersion and line broadening (fig. S4-31 and tab. S2, 3).

The first compound to be studied was chitinopeptin A. The analysis of the NMR spectra revealed the presence of several canonical amino acids (1 Thr, $1 \mathrm{Ala}, 1 \mathrm{Ile}, 1 \mathrm{Ser}, 1 \mathrm{Lys}$, and $2 \mathrm{Leu}$ ), and several hydroxylated amino acids ( $3 \beta$-OH Asp, $1 \beta$-OH Phe, $1 \beta$-OH Ile). In addition, one $N$-methyl $\mathrm{Val}$ and one 2,3-diaminopropionic acid (Dap) moiety could be assigned. The sequence of the amino acids was established by correlations in the ROESY $\left(\mathrm{NH}_{\mathrm{i}} / \mathrm{NH}_{\mathrm{i}+1}, \mathrm{NH}_{\mathrm{i}+1} / \mathrm{H \alpha}_{\mathrm{i}}\right)$ and $\mathrm{HMBC}$ spectrum $\left(\mathrm{C}^{\prime}{ }_{\mathrm{i}} / \mathrm{NH}_{\mathrm{i}+1}\right)$. The formation of a cyclic peptide was indicated by the ${ }^{1} \mathrm{H}$-chemical shift of the $\beta$-proton of the $\mathrm{Thr}$ residue in position 2 $(5.23 \mathrm{ppm})$ and the correlation in the HMBC spectrum between the carbonyl carbon of the $C$-terminal $\beta$-OH Ile (position 14) and the $\beta$-proton of the Thr residue.

Aside from the amino acids, a modified fatty acid residue was identified which could be described as 2,9-dimethyl-3-amino decanoic acid. Correlations in the HMBC spectrum between the carboxyl carbon $(\mathrm{C} 1)$ and the $N$-methyl group of the $N$-methyl Val proved its position at the $N$-terminus of the peptide. The structure of chitinopeptin B was almost identical to the structure of $\mathbf{1}$. The only difference was the substitution of the 2,9-dimethyl3-amino decanoic acid by 3-amino-9-methyl decanoic acid.

CLPs 3 and 4 were isolated as a 5:4 mixture of two components. One of the main differe nces compared to the structures above was an additional Dap residue, which was inserted between the fatty acid moiety and $N$-methyl Val. Both components contain an Ile instead of a Leu (1 and 2) at position 10. Furthermore, the 2,9-dimethyl-3-amino decanoic acid is replaced by a 3-hydroxy-9-methyl decanoic acid. The two components 3 and $\mathbf{4}$ differ in the constitution of the Dap in position 9. In one component (4) the peptide bond between the $\alpha$ amino function and the carbonyl group of Lys is formed, while in the other component (3), the $\beta$-amino group (side chain) is connected to the carbonyl group of Lys. The same pair of structures as for CLPs 3 and $\mathbf{4}$ is obtained in the case of CLPs $\mathbf{5}$ and $\mathbf{6}$. In contrast to the previous ones, both components contain a Val in position 10 instead of Leu or Ile, respectively.

The absolute stereochemistry of the amino acids in the CLPs was determined by using advanced Marfey's Analysis(71). Comparison of the RTs with (commercially available) reference amino acids allowed identification of 9 out of 14 (1 and 2) and 10 out of 15 amino acids (3-6), respectively. RTs of $N$-methyl-L-Val, D-allo-Thr, D-Ala, D-allo-Ile (2x 3 and 4), L-Ser, D-Lys, L-Dap (2x for 3-6), D-Leu (only 1 and 2), L-Leu, and D-Val (only 5 and 6) matched the reference ones. Assigning L- as well as D-leucine within structures $\mathbf{1}$ and $\mathbf{2}$ to position 13 and 10, respectively, was possible because position 10 was the only variable position in all six depsipeptides. Either Ile (3 and 4) or Val (5 and $\mathbf{6})$ were identified at this position with all amino acids having D-configuration. Therefore, it can be assumed that DLeu is present at position 10 in $\mathbf{1}$ and $\mathbf{2}$ (fig. S32, 33).

Contrary to data base results, nearly all of the $\beta$-hydroxyamino acids could not be delivered from commercial suppliers. Therefore, authentic samples of these amino acids or suitable precursors were synthesized utilizing modified literature known procedures. All four stereoisomers of $\beta$-hydroxyaspartic acid were obtained from (-)-dibenzyl D-tartrate or (+)dibenzyl L-tartrate, respectively, according to a procedure described by Breuning et al(72). While the anti-isomers are directly accessible, the syn-isomers were obtained by selective base-induced epimerization of the azido-intermediates and separation of the two isomers by HPLC. Cbz-protected L-isomers of the $\beta$-hydroxyphenylalanines and $\beta$-hydroxyisoleucines were synthesized starting from an orthoester protected L-serine aldehyde, initially described by Blaskovich and Lajoie(73-75). To cover the corresponding D-isomers for analytical purposes, racemic samples of the amino acids were produced by racemization of the 
orthoester protected L-serine aldehyde by simple chromatography on silica(73). Advanced Marfey's Analysis determined (2S,3S)-3-hydroxyaspartic acid, (2S,3R)-3hydroxyphenylalanine and $(2 S, 3 R)$-3-hydroxyisoleucine as the absolute stereochemistry of $\beta$-hydroxyamino acids for all six CLPs (fig. S34-36).

To the best of our knowledge, these represent the first CLPs described from the genus Chitinophaga and after the recently described isopedopeptins(12) the second CLP class of the entire phylum. They contain a high number of non-proteinogenic amino acids (i.e., 12 of 14 amino acids in $\mathbf{1}$ and 2, and 13 of 15 in 3-6). Beta-hydroxylations of Asp, Phe and Ile are the most abundant modifications and $N$-methyl Val and Dap are incorporated into the peptide backbone. Furthermore, during LC-MS analysis, a mass shift of $52.908 \mathrm{Da}$ accompanied with an emerging UV maximum at $310 \mathrm{~nm}$, as well as a shift in RT was observed for 1-6. This traced back to the coordination of the compounds to iron impurities during LC-analysis, which was confirmed by the addition of $\mathrm{Fe}$ (III)-citrate to the compounds prior to LC-MS analysis (fig. S37). We postulated that the RT shift is due to a conformational change and altered polarities as a consequence of iron complexation. Iron coordination is a known feature of siderophores, produced by bacteria upon low iron stress(76). To investigate the impact of iron on the CLPs production, C. eiseniae was cultured in 3018 medium supplemented with different iron concentrations. However, the overall production of CLPs $\mathbf{1}$ and $\mathbf{2}$ and their iron complexes was not repressed by increased iron levels, contrasting the iron-responsive productivity of classical siderophores (fig. S38).

Chitinopeptins A-D were tested against Gram-negative and Gram-positive bacteria, as well as against three filamentous fungi and $C$. albicans (tab. S4). For all tested CLPs activity was observed against $M$. catarrhalis ATCC 25238 and B. subtilis DSM 10, exhibiting MICs of down to $2 \mu \mathrm{g} / \mathrm{mL}$. The tetradecalipodepsipeptides 1 and 2 exhibited activity at $4-8 \mu \mathrm{g} / \mathrm{mL}$ against $C$. albicans FH2173, while the pentadecalipodepsipeptides exhibited MICs of only $16 \mu \mathrm{g} / \mathrm{mL}$. Screenings against filamentous fungi revealed MICs of $16 \mu \mathrm{g} / \mathrm{mL}$ against $Z$. tritici MUCL45407, while no activity was observed against A. flavus ATCC 9170 and $F$. oxysporum ATCC 7601. To investigate the impact of iron-binding on the bioactivity, 1 was tested as a representative of this class also in its iron complexed form confirmed by LC-MS analysis. This revealed that the bioactive potency of the iron complex is reduced in comparison to its iron free form, although not completely suppressed.

\section{BGCs corresponding to chitinopeptins}

In order to identify the BGCs encoding the chitinopeptins' biosynthesis, we scanned the genomes of $C$. eiseniae (FUWZ01.1) and $C$. flava (QFFJ01.1) for NRPS-type BGCs matching the structural features of the molecules. The number and predicted substrate specificity of the A-Domains, the overall composition of the NRPS assembly line, as well as precursor supply and post-assembly modifications were taken into account. We identified the BGCs in $C$. eiseniae and $C$. flava congruent to the CLP structure in each case (Fig. 5A). Furthermore, the positioning of all epimerization domains within the detected NRPS genes, encoding the conversion of L- to D-amino acids, is in agreement with the determined stereochemistry of the molecules. Thereby, the domains are classically embedded in the NRPS assembly lines. No racemase(s) encoded in trans or C domains catalyzing the conversion of amino acids, are observed as it is the case e.g. in the BGC of the stechlisins, CLPs produced by Pseudomonas sp.(77).

Retroperspective evaluation of our BiG-SCAPE network revealed a GCF comprising these BGCs from C. eiseniae and C.flava. Manual inspection confirmed two further related BGCs within the genomes of $C$. oryziterrae JCM16595 (WRXO01.1) and C. niastensis DSM 24859 (PYAW01.1) (Fig. 5B). Besides the structural NRPS genes, further genes are conserved between all four BGCs, predicted to encode an ATP-binding cassette (ABC) 
transporter, a transcription factor, a S8 family serine peptidase, a thioester reductase domain and a metal $\beta$-lactamase fold metallo-hydrolase. The biosynthesis of these CLPs requires a $\beta$-hydroxylation tailoring reaction of the precursor Asp, Phe and Ile. This structural feature is also present in chloramphenicol and its biosynthesis was shown to be catalyzed by the diiron-monooxygenase $\mathrm{CmlA}$ that catalyzes substrate hydroxylation by dioxygen activation. CmlA coordinates two metal ions within a His-X-His-X-Asp-His motif and possesses a thioester reductase domain(78). Both features are also present in all four detected BGCs, encoded by two separate genes annotated as thioester reductase domain and metal $\beta$-lactamase fold metallo-hydrolase (gene 3 and 4) (Fig. 5B and tab. S5). Moreover, all four BGCs contain genes with sequence similarity to $\operatorname{sbn} A$ and $\operatorname{sbn} B$, encoding enzymes that catalyze the synthesis of the non-proteinogenic amino acid $\operatorname{Dap}(79,80)(\operatorname{tab}$. S6). The presence of genes encoding for supply with precursor amino acid(s) is a common feature in BGCs corresponding to NPs described in the Firmicutes and Actinobacteria phyla(81-86). Interestingly, within the BGC of $C$. eiseniae a $\operatorname{sbnB}$ homologue is missing. To rule out an error during genome sequencing, assembly and annotation, we amplified the respective section by PCR and confirmed the published genome sequence. However, not encoded in cis, C. eiseniae carries further $\operatorname{sbn} A$ and $\operatorname{sbn} B$ homologues. These are encoded in other NRPS-type BGCs (FUWZ01000006, location: 555,550-557,479 and FUWZ01000004, location: 273,583-275,522) and potentially function in trans to compensate the absence of the gene within the chitinopeptin A and B BGC. In general, we observed that the Dap subcluster $\operatorname{sbn} A / B$ is an abundant genetic feature of Bacteroidetes secondary metabolis $m$, present in many BGCs. This is not restricted to Chitinophaga, but expanded to different classes and in consequence, a structural feature of Bacteroidetes NPs predictively found with high frequency. Indeed, Dap moieties are present in the known antibacterial Bacteroidetes compounds isopedopeptins(12) and TAN-1057 A-D(43).

Considering the composition of the structural NRPS genes in terms of A domain number as well as predicted A domain substrate specificity $(87)$, we propose that $C$. oryziterrae and $C$. niastensis carry the potential to provide additional structural variety to the chitinopeptin class (tab. S7). The NRPS gene of $C$. niastensis encodes 16 A-domain-containing modules, predictably producing hexadecapeptides. Furthermore, an initial PKSI module replaces the C-starter domains present in the three other BGCs of this GCF. This points toward to an attachment of carboxylic acid residues to the peptide scaffold, resulting in further compound diversification. While $C$. oryziterrae was not available for cultivation, we inspected the extracts of $C$. niastensis for putative further CLPs. Indeed, although only detected in traces, possible products could be identified based on comparable RTs and isotope patterns with $\mathrm{m} / \mathrm{z}$ of $680.9817[\mathrm{M}+3 \mathrm{H}]^{3+}$ and $685.6545[\mathrm{M}+3 \mathrm{H}]^{3+}$ (fig. S39).

\section{Discussion}

Mining microbes for their secondary/specialized metabolites continues to be an essential and valuable part of drug discovery pipelines. With the constant rediscovery of already known NPs, the past decades are representative for the limitations of classical approaches focusing on the most talented producer taxa that only represent a limited phylogenetic space of the bacterial kingdom(34). However, the microbial diversity is growing with each new phylum added to the tree of life(20). In parallel, rapid improvements of resolution and accuracy allowed to apply technologically more complex metabolomics- and genomicsguided methods to source nature $(24,25,31,33)$. Genomics-guided methods predict the strains phenotype and their theoretical ability to produce compounds, based on identified BGCs and their consecutive prioritization(23). Complementary omic-technologies, especially metabolomics enable the sophisticated characterization of the strains' 
chemotypes, the actually detected metabolites. Standalone or applied in sequel, both technologies already made a strong impact on the field of NPs. (Semi)auto matized combinations of genomics and metabolomics are under development and aim to establish a direct link between the biosynthetic potential of microbial strains and their actually expressed metabolites $(29,33,88)$. The basis for this was built over the last years and gave evidence that those tools are as powerful as their underlying training datasets(89).

In future, these approaches will likely support the field by promoting reduction of rediscovery rates and in turn allowing efficient sample prioritization with respect to the target molecules of the respective studies. Until today, these approaches were primarily applied on big datasets from well-known NP producing taxa such as Actinobacteria or Myxobaceria(29, 30). These studies enabled technology development, while directly expanding the overall knowledge on the still available chemical space of these taxa as well as the identification of novel secondary metabolites or derivatives of known ones. Here, we translated this approach of application of complementary omics-technologies for the first time to the yet underexplored Bacteroidetes phylum. The comprehensive computational analysis of publicly available Bacteroidetes genomes revealed the Chitinophaga as most promising genus in terms of NP production capabilities. The genome-wide evaluation of 47 Chitinophaga strains showed the presence of 15.7 BGCs on average, with a minimum of six and a maximum of 31 BGC. Although the number of detected BGCs is strongly dependent on the utilized bioinformatics tools and chosen settings, the most talented Chitinophaga competed with the BGC loads of Actinobacteria(90). Moreover, the variation of BGC amount within a genus is a known phenomenon and also observed e.g. in the Amycolatopsis genus(91).

Today, the Dictionary of Natural Products (DNP)(92) (01/2021) contains only 14 entries ascribed to the genus Chitinophaga. This does not reflect their predicted genetic potential, since e.g. for the genus Streptomyces 8,969 chemical entities are deposited. The extrapolation of the approximately doubled BGC load of Streptomyces (25-70 BGCs)(50) results in $~ 650$ times more chemical entries, a theoretical gap that justifies efforts to investigate the metabolic repertoire of the genus Chitinophaga. Our metabolomics analysis of 25 Chitinophaga strains confirmed the genomic and database-based prediction by empirical data. The identified chemical space is represented by more than 1,000 unique and unknown candidates that could not even been associated to any microbial NP known today. The uniqueness and value of this dataset compares with data sets gathered by metabolomics studies of well-known NP producer taxa. Although comparability of such extensive approaches is challenging due to different dataset size, composition as well as technical devices and settings, the detection of bucket/metabolite numbers per strain in our Chitinophaga strain set is in similar range than the ones described for different Myxobacteria genera(32). One striking difference however, is the lack of dereplicated known metabolites, which can be explained by their underrepresentation in all reference databases utilized in this study for data categorization. This is likely due to the fact that investigations of the genus Chitinophaga and the Bacteroidetes phylum itself have just started.

In conclusion, our analys is reveals that the Bacteroidetes phylum has an overall distinct metabolic repertoire compared to classical NP producer taxa as indicated by the nonconnatural taxonomy relation of their GCFs. Eventually, our combinatorial approach in utilizing genomics and metabolomics facilitated the genus and strain prioritization and paved the way for the discovery of the chitinopeptins A-D. These NRPS-assembled CLPs exhibit primarily activity against Candida albicans and were found to coordinate iron. Binding of metal ions is a feature also described for other CLPs such as pseudofactin II, 
which displays an increased antimicrobial activity upon metal-coordination due to disruption of the cytoplasmic membrane in its chelated state(93). Incidences of fungal infections, including candidiasis caused by Candida species are increasing(94). Currently, clinically used antifungals are limited to four classes: azoles, polyenes, echinocandins, and pyrimidine analogs. Associated with occurrence and spreading of resistances, this leads to alarmingly decreased treatment success and increased possibilities of fatal outcomes(95). This development clearly demands counteraction. One possible way to do is the constant discovery of novel NPs with antifungal activities. The chitinopeptins share promising properties with the underutilized as well as promising NP group of CLPs that exhibit an intrinsic antifungal activity. Representatives thereof are e.g. CLPs of the surfactin or iturin(96) and fengycin(15) class with similar activities reported against Candida. They are under investigation to be or have already been commercialized as biocontrol agents, food preservatives and in biomedical fields $(16,97)$. Mode of action studies for both classes suggest a membrane interaction and leakage effects up to pore-forming properties(98-101). Further studies are necessary to elucidate the functional role and the activity profile of the chitinopeptins.

\section{Materials and Methods}

\section{Genomic data processing.}

Genomic data were collected until 01/2021. To that date, almost all publically, complete and correctly annotated genomes in addition with some WGS projects of strains of interest of the phylum Bacteroidetes (600 genomes in total, tab. S8) were analyzed using antiSMASH 5.0(49) and BiG-SCAPE v1.0.0(26). All Bacteroidetes genome sequences used in this study were downloaded from the National Center for Biotechnology Information (NCBI) database or the Department of Energy (DOE) Joint Genome Institute - Integrated Microbial Genomes \& Microbiomes (JGI IMG) database(102, 103). The dataset consists of all six phylogenetic classes with the following distribution: Bacteroidia 18\% $(\mathrm{n}=108)$, Chitinophagia 13.7\% $(\mathrm{n}=82)$, Cytophagia $11.5 \%(\mathrm{n}=69)$, Flavobacteriia $50.2 \%(\mathrm{n}=301)$, Saprospiria 0.3\% $(\mathrm{n}=2)$, Sphingobacteriia $6.3 \%(\mathrm{n}=37)$.

\section{Phylogenetic tree reconstruction.}

For the phylogenetic tree of the complete Bacteroidetes phylum we aligned the extracted $16 S$ rRNA genes from each genome assembly using MAFFT(104). A maximum likelihood phylogeny was build based on the $16 S$ rRNA genes using the program RAxML v.8.2.11(105) with a general time reversible (GTR) nucleotide substitution model(106) and 1000 bootstrap replicates. We used the Interactive Tree of Life [iToL v4](107) to visualize the phylogenetic tree. The labels of each branch are color coded by class level. A strip dataset represents the genome size of each strain, from light grey for small sizes to black for large genomes. A multi value bar chart placed around the tree displays the total BGC amount of each strain, in parallel highlighting the specific amount of NRPS, PKS and the hybrid BGCs of both types. Partial BGCs on contigs $<10 \mathrm{~kb}$ of whole genome shotgun (WGS) projects are not included in this analysis.

The phylogenetic tree of the genus Chitinophaga is based on a Clustal W alignment(108) of available $16 \mathrm{~S}$ rRNA gene sequences of 25 Chitinophaga strains available for cultivation. The tree was calculated using MEGA v7.0.26 with the maximum-likelihood method and GTR-Gamma model(109). Percentage on the tree branches indicate values of 1,000 bootstrap replicates with a bootstrap support of more than $50 \%$. The tree is drawn to scale, with branch lengths measured in the number of substitutions per site.

\section{BiG-SCAPE-CORASON analysis.}

Individual .gb files were processed using antiSMASH 5.0, including the ClusterFinder border prediction algorithm to automatically trim BGCs where gene cluster borders were 
possible to predict(23, 49). The "hybrids" mode of BiG-SCAPE v1.0.0(26), which allows BGCs with mixed annotations to be analyzed together, was enabled. Several cutoffs of 0.1 to 0.9 were tested. After manual inspection the network with a cutoff of 0.6 was visualized and annotated using Cytoscape v3.6.0(110).

\section{Mass spectrometric analysis.}

A quadrupole time-of-flight spectrometer (LC-QTOF maXis II, Bruker Daltonik) equipped with an electrospray ionization source in line with an Agilent 1290 infinity LC system (Agilent) was used for all UHPLC-QTOF-UHR-MS and MS/MS measurements. C18 RPUHPLC [ACQUITY UPLC BEH C18 column (130 $\AA, 1.7 \mu \mathrm{m}, 2.1$ x $100 \mathrm{~mm})$ ] was performed at $45{ }^{\circ} \mathrm{C}$ with the following linear gradient: 0 min: $95 \% \mathrm{~A} ; 0.30 \mathrm{~min}$ : $95 \% \mathrm{~A}$; $18.00 \mathrm{~min}: 4.75 \% \mathrm{~A} ; 18.10 \mathrm{~min}: 0 \% \mathrm{~A} ; 22.50 \mathrm{~min}: 0 \% \mathrm{~A} ; 22.60 \mathrm{~min}: 95 \% \mathrm{~A} ; 25.00 \mathrm{~min}$ : 95\% A (A: $\mathrm{H}_{2} \mathrm{O}, 0.1 \% \mathrm{HCOOH}$; $\mathrm{B}: \mathrm{CH}_{3} \mathrm{CN}, 0.1 \% \mathrm{HCOOH}$; flow rate: $\left.0.6 \mathrm{~mL} / \mathrm{min}\right)$. Mass spectral data were acquired using a 50 to $2000 \mathrm{~m} / \mathrm{z}$ scan range at $1 \mathrm{~Hz}$ scan rate. MS/MS experiments were performed with $6 \mathrm{~Hz}$ and the top five most intense ions in each full MS spectrum were targeted for fragmentation by higher-energy collisional dissociation at $25 \mathrm{eV}$ or $55 \mathrm{eV}$ using $\mathrm{N}_{2}$ at $10^{-2}$ mbar. Precursors were excluded after 2 spectra, released after $0.5 \mathrm{~min}$ and reconsidered if the intensity of an excluded precursor increased by factor 1.5 or more.

\section{Chemotype-barcoding matrix.}

Raw data processing: Raw data processing was performed with DataAnalysis 4.4 (Bruker) using recalibration with sodium formate. RecalculateLinespectra with two thresholds (10.000 and 20.000) and subsequent FindMolecularFeatures (0.5 to $25 \mathrm{~min}, \mathrm{~S} / \mathrm{N}=0$, minimal compound length $=8$ spectra, smoothing width $=2$, correlation coefficient threshold $=0.7$ ) was performed. Bucketing was performed using ProfileAnalysis 2.3 (Bruker; 30 to $1080 \mathrm{~s}, 100$ to $6000 \mathrm{~m} / \mathrm{z}$, Advanced Bucketing with $24 \mathrm{~s} 5 \mathrm{ppm}$, no transformation, Bucketing basis $=\mathrm{H}^{+}$).

Data management: The complete dataset was copied and processed with both line spectra thresholds (10k and 20k). Bucketing was performed on both sets at the same time, resulting in one table containing all buckets deemed identical for every sample (under both thresholds). This table was subsequently curated: Buckets were only deemed present, if they were detected in the 20k samples (4707 buckets did not meet this criteria and were deleted). To avoid 'uniqueness' due to compounds being just above the detection threshold, entries from the $10 \mathrm{k}$ set were used for these buckets, the $20 \mathrm{k}$ entries were deleted. 80 buckets were not filled in this table and were subsequently deleted, resulting in a final table of 278 samples with 4188 buckets.

For preparation of the barcode, for each strain (and combined control) and fermentation length (4 and 7 days) was analyzed, how often each bucket was present. Since media controls were repeated several times (instead of once per strain/duration combination), they were combined first. Each bucket present in one of the control/time/media combinations was deemed present.

Classifications were 'more than one' for buckets present in two or more media; 'media $\mathrm{X}$ ' for buckets present in only one media and 'none' for all not present at that strain/duration combination. This table is the input table for the barcode matrix. For each strain was calculated, if a bucket was present in at least one of its media/duration combinations. Subsequently it was calculated, in how many strains (excluding media controls) each bucket was present.

To achieve a readable representation, the buckets in the input table were sorted (after transformation/rotation): 1. (alphabetically) for each sample (for defragmentation of media), 2. (decreasing) for the number of strains in which the bucket is present, and 3. presence in 
any of the control conditions. Visualization was performed with the R-script deposited in the SI.

\section{Cultivation and screening conditions.}

Bacterial strains and culture conditions: All 25 Chitinophaga strains used for this study (tab. S1) were purchased from Deutsche Sammlung von Mikroorganismen und Zellkulturen (DMSZ) and Korean Collection for Type Cultures (KCTC). Strains were inoculated in $50 \mathrm{~mL}$ R2A(111) (HiMedia Laboratories, LLC.) in $300 \mathrm{~mL}$ flasks and incubated for 3 days, then $2 \%(\mathrm{v} / \mathrm{v})$ culture volume was used to inoculate the main cultures $(50 \mathrm{~mL}$ media/300 mL flask), incubated at $28{ }^{\circ} \mathrm{C}$ with agitation at $180 \mathrm{rpm}$ for 4 or 7 days. $\mathrm{R} 2 \mathrm{~A}, 3018(1 \mathrm{~g} / \mathrm{L}$ yeast extract, $5 \mathrm{~g} / \mathrm{L}$ casitone, $\mathrm{pH} 7.0,24 \mathrm{mM} \mathrm{N}$-acetylglucosamine added after autoclaving), 5294 $(10 \mathrm{~g} / \mathrm{L}$ soluble starch, $10 \mathrm{~g} / \mathrm{L}$ glucose, $10 \mathrm{~g} / \mathrm{L}$ glycerol 99\%, $2.5 \mathrm{~g} / \mathrm{L}$ liquid corn steep, $5 \mathrm{~g} / \mathrm{L}$ peptone, $2 \mathrm{~g} / \mathrm{L}$ yeast extract, $1 \mathrm{~g} / \mathrm{L} \mathrm{NaCl}, 3 \mathrm{~g} / \mathrm{L} \mathrm{CaCO} 3, \mathrm{pH}$ 7.2), $3021(10 \mathrm{~g} / \mathrm{L}$ glucose, $10 \mathrm{~g} / \mathrm{L}$ chitin, $5 \mathrm{~g} / \mathrm{L}$ soy flour, $5 \mathrm{~g} / \mathrm{L}$ casein peptone, $\mathrm{pH} 7.0)$ and $5065(15 \mathrm{~g} / \mathrm{L}$ soluble starch, $10 \mathrm{~g} / \mathrm{L}$ glucose, $10 \mathrm{~g} / \mathrm{L}$ soy flour, $1 \mathrm{~g} / \mathrm{L}$ yeast extract, $0.1 \mathrm{~g} / \mathrm{L} \mathrm{K} 2 \mathrm{HPO} 4,3 \mathrm{~g} / \mathrm{L} \mathrm{NaCl}, \mathrm{pH}$ 7.4) supplemented with $1 \mathrm{~mL} / \mathrm{L}$ SL-10 trace element solution(112) and $3 \mathrm{~mL} / \mathrm{L}$ vitamin solution (added after autoclaving, VL-55 medium, DSMZ) were used as main culture media.

Screening conditions: Freeze-dried cultures were extracted with $40 \mathrm{~mL} \mathrm{MeOH}$, dried and resuspended in $1 \mathrm{~mL} \mathrm{MeOH}$ to generate $50 \mathrm{x}$ concentrated extracts, partially used for extract analysis by ultra high performance liquid chromatography-high resolution mass spectrometry (UHPLC-UHR-MS, details in MS analysis part). An aliquot of $150 \mu \mathrm{L}$ of each extract was dried again and resuspended in $75 \mu \mathrm{L}$ DMSO, resulting in 100x concentrated extracts. Four concentrations ( $1 \mathrm{x}, 0.5 \mathrm{x}$ and $0.25 \mathrm{x}$ twice) of each extract were screened in 384 well plate format ( $20 \mu \mathrm{L}$ assay volume) supported by liquid handling robots (Analytik Jena CyBio Well 96/384 CYBIO SW-CYBI, Thermo scientific Matrix Wellmate and Multidrop). Assay plates were inoculated with $20.000 \mathrm{CFUs} / \mathrm{mL}$ from overnight precultures $(100 \mathrm{~mL}$ flasks filled with $30 \mathrm{~mL}$ cation adjusted Mueller Hinton II Broth (MHIIB, BD Difco), $37^{\circ} \mathrm{C}$ ) diluted in MHIIB of E. coli ATCC 35218, E. coli ATCC $25922 \Delta \mathrm{TolC}$, P. aeruginosa ATCC 27853, K. pneumoniae ATCC 13883 or S. aureus ATCC 25923 and incubated at $37^{\circ} \mathrm{C}$ overnight. The protocol was adapted to an inoculum of 100.000 CFUs $/ \mathrm{mL}$ for M. smegmatis ATCC 607, C. albicans FH2173, and 50.000 spores $/ \mathrm{mL}$ from a spore solution for A. flavus ATCC 9170 . Brain heart infusion (BHI) broth supplemented with $1 \%(\mathrm{v} / \mathrm{v})$ Tween 80 for M. smegmatis and MHIIB for $A$. flavus and C. albicans were used. Pre-cultures and main-cultures were incubated at $37^{\circ} \mathrm{C}$ for two days, except the pre-culture of C. albicans was incubated at $28^{\circ} \mathrm{C}$. All assay plates and pre-cultures were incubated with agitation at $180 \mathrm{rpm}$ and controlled humidity $(80 \%$ $\mathrm{RH})$ to prevent evaporation. Active extracts were defined as $>80 \%$ growth inhibition compared to the controls. Optical density $(600 \mathrm{~nm})$ or for A. flavus, C. albicans and $M$. smegmatis a quantitative ATP assay (BacTiter-Glo, Promega) based on the resulting relative light units, both measured with a LUMIstar OPTIMA Microplate Luminometer (BMG LABTECH), was used according to manufactures protocol to detect growth inhibition. UHPLC-UHR-ESI-MS guided fractionation helped to identify and dereplicate possible active molecules within crude extracts.

Strain fermentation and purification of chitinopeptins.

C. eiseniae DSM 22224 and C. flava KCTC 62435 were inoculated from plate (R2A) in $300 \mathrm{~mL}$ Erlenmeyer flasks filled with $100 \mathrm{~mL}$ R2A and incubated at $28^{\circ} \mathrm{C}$ with agitation at $180 \mathrm{rpm}$ for 3 days. Followed by $20 \mathrm{~L}$ fermentations (separated in $500 \mathrm{~mL}$ culture volume per 2 L flasks) in 3018-medium inoculated with $2 \%(\mathrm{v} / \mathrm{v})$ pre-culture, incubated under the same conditions for 4 days and subsequently freeze-dried. Dried cultures were extracted with one-time culture volume $\mathrm{MeOH}$. The extracts were evaporated to dryness using rotary evaporation under reduced pressure, resuspened in $3 \mathrm{~L}$ of $10 \% \mathrm{MeOH} / \mathrm{H}_{2} \mathrm{O}$ and loaded onto 
a XAD16N column (1 L bed volume). Step-wise elution with 10\%, 40\%, 60\%, 80\%, and $100 \% \mathrm{MeOH}$ (two-times bed volume each) was performed. The 80 and $100 \%$ fractions containing chitinopeptins were subjected to preparative followed by semi-preparative reverse-phase high-performance liquid chromatography (RP-HPLC) on C18 columns (preparative HPLC: Synergi $4 \mu \mathrm{m}$ Fusion-RP $80 \AA$ (250 x $21.2 \mathrm{~mm})$; semi preparativeHPLC: Synergi $4 \mu \mathrm{m}$ Fusion-RP $80 \AA$ (250 x $10 \mathrm{~mm})$, mobile phase: $\mathrm{CH}_{3} \mathrm{CN} / \mathrm{H}_{2} \mathrm{O}+0.1 \%$ $\mathrm{HCOOH}$ ) using linear gradients of 5-95\% organic in $40 \mathrm{~min}$. Final purification was achieved using UHPLC on a ACQUITY UPLC BEH C18 column (130 А, $1.7 \mu \mathrm{m}, 100 \mathrm{x}$ $2.1 \mathrm{~mm}$ ) with the same mobile phase and a linear gradient of $30-60 \%$ organic in $18 \mathrm{~min}$. After each step, fractions containing chitinopeptins were evaporated to dryness using a high performance evaporator (Genevac HT-12).

Chitinopeptin A (1). White, amorphous powder; $[\alpha]_{\mathrm{D}}^{20}=-27.6^{\circ}\left(c=0.11, \mathrm{CH}_{3} \mathrm{OH}\right)$; LC-UV $\left[\left(\mathrm{CH}_{3} \mathrm{CN}\right.\right.$ in $\left.\left.\mathrm{H}_{2} \mathrm{O}+0.1 \% \mathrm{HCOOH}\right)\right] \lambda \max 220 \mathrm{~nm} ;{ }^{1} \mathrm{H}-\mathrm{NMR}$ and ${ }^{13} \mathrm{C}-\mathrm{NMR}$ data, see Supplementary Table 2 and 3; UHR-MS (ESI-TOF) $m / z[\mathrm{M}+\mathrm{H}]^{+}$calcd for $\mathrm{C}_{82} \mathrm{H}_{138} \mathrm{~N}_{17} \mathrm{O}_{28}$ 1809.0037, found 1809.0052.

Chitinopeptin B (2). White, amorphous powder; $[\alpha]_{\mathrm{D}}^{20}=-21.0^{\circ}\left(c=0.16, \mathrm{CH}_{3} \mathrm{OH}\right)$; LC-UV $\left[\left(\mathrm{CH}_{3} \mathrm{CN}\right.\right.$ in $\left.\left.\mathrm{H}_{2} \mathrm{O}+0.1 \% \mathrm{HCOOH}\right)\right] \lambda \max 222 \mathrm{~nm} ;{ }^{1} \mathrm{H}-\mathrm{NMR}$ and ${ }^{13} \mathrm{C}-\mathrm{NMR}$ data, see Supplementary Table 2 and 3; UHR-MS (ESI-TOF) $m / z[\mathrm{M}+\mathrm{H}]^{+}$calcd for $\mathrm{C}_{81} \mathrm{H}_{136} \mathrm{~N}_{17} \mathrm{O}_{28}$ 1794.9880, found 1794.9907.

Chitinopeptin $\mathrm{C} 1+\mathrm{C} 2$ (3 and 4). Slightly yellowish, amorphous powder; LC-UV [ $\left(\mathrm{CH}_{3} \mathrm{CN}\right.$ in $\left.\left.\mathrm{H}_{2} \mathrm{O}+0.1 \% \mathrm{HCOOH}\right)\right] \lambda \max 219 \mathrm{~nm} ;{ }^{1} \mathrm{H}-\mathrm{NMR}$ and ${ }^{13} \mathrm{C}-\mathrm{NMR}$ data, see Supplementary Table 2 and 3; UHR-MS (ESI-TOF) $m / z$ [M+H] ${ }^{+}$calcd for $\mathrm{C}_{84} \mathrm{H}_{141} \mathrm{~N}_{18} \mathrm{O}_{30} 1882.0061$, found 1882.0177.

Chitinopeptin D1+D2 (5 and 6). Slightly yellowish, amorphous powder; LC-UV [ $\left(\mathrm{CH}_{3} \mathrm{CN}\right.$ in $\left.\left.\mathrm{H}_{2} \mathrm{O}+0.1 \% \mathrm{HCOOH}\right)\right] \lambda \max 221 \mathrm{~nm} ;{ }^{1} \mathrm{H}-\mathrm{NMR}$ and ${ }^{13} \mathrm{C}-\mathrm{NMR}$ data, see Supplementary Table 2 and 3; UHR-MS (ESI-TOF) $m / z$ [M+H] $]^{+}$calcd for $\mathrm{C}_{83} \mathrm{H}_{139} \mathrm{~N}_{18} \mathrm{O}_{30} 1867.9905$, found 1868.0059 .

\section{Structure elucidation}

NMR studies: NMR spectra of chitinopeptin A (1) were acquired on a Bruker AVANCE 700 spectrometer operating at a proton frequency of $700.13 \mathrm{MHz}$ and a ${ }^{13} \mathrm{C}$-carbon frequency of $176.05 \mathrm{MHz}$. NMR spectra of the remaining compounds were recorded on a Bruker AVANCE 500 spectrometer operating at a proton frequency of $500.30 \mathrm{MHz}$ and a ${ }^{13} \mathrm{C}$-carbon frequency of $125.82 \mathrm{MHz}$. Both instruments were equipped with a $5 \mathrm{~mm}$ TCI cryo probe. For structure elucidation and assignment of proton and carbon resonances 1D${ }^{1} \mathrm{H}$, 1D- ${ }^{13} \mathrm{C}$, DQF-COSY, TOCSY (mixing time $80 \mathrm{~ms}$ ), ROESY (mixing time $150 \mathrm{~ms}$ ), multiplicity edited-HSQC, and HMBC spectra were acquired.

Homonuclear experiments (1D- ${ }^{1} \mathrm{H}$, DQF-COSY, TOCSY, ROESY) were acquired in a mixture of $\mathrm{H}_{2} \mathrm{O}$ and $\mathrm{CD}_{3} \mathrm{CN}$ in a ratio of $1: 1$. 1D- ${ }^{13} \mathrm{C}$, HSQC and $\mathrm{HMBC}$ spectra were acquired in a mixture of $\mathrm{D}_{2} \mathrm{O}$ and $\mathrm{CD}_{3} \mathrm{CN}$ in a ratio of $1: 1$. In the case of CLP 1 the HMBC spectrum has also been acquired in $\mathrm{H}_{2} \mathrm{O} / \mathrm{CD}_{3} \mathrm{CN} .{ }^{1} \mathrm{H}$-chemical shifts were referenced to sodium-3-(Trimethylsilyl)propionate-2,2,3,3- $\mathrm{d}_{4} .{ }^{13} \mathrm{C}$-chemical shifts were referenced to the solvent signal $\left(\mathrm{CD}_{3} \mathrm{CN},{ }^{13} \mathrm{C}: 1.30 \mathrm{ppm}\right)$.

Advanced Marfey's Analysis: The absolute configuration of all amino acids was determined by derivatization using Marfey's reagent(71). Stock solutions of amino acid standards $\left(50 \mathrm{mM}\right.$ in $\left.\mathrm{H}_{2} \mathrm{O}\right), \mathrm{NaHCO}_{3}\left(1 \mathrm{M}\right.$ in $\left.\mathrm{H}_{2} \mathrm{O}\right)$, and $N_{\alpha}$-(2,4-dinitro-5-fluorophenyl)-Lvalinamide (L-FDVA, $70 \mathrm{mM}$ in acetone) were prepared. Commercially available and synthesized standards were derivatized using molar ratios of amino acid to FDVA and $\mathrm{NaHCO}_{3}(1 / 1.4 / 8)$. After stirring at $40{ }^{\circ} \mathrm{C}$ for $3 \mathrm{~h}, 1 \mathrm{M} \mathrm{HCl}$ was added to obtain concentration of $170 \mathrm{mM}$ to end the reaction. Samples were subsequently evaporated to dryness and dissolved in DMSO (final concentration $50 \mathrm{mM}$ ). L- and D-amino acids were 
analyzed separately using C18 RP-UHPLC-MS $\left(\mathrm{A}: \mathrm{H}_{2} \mathrm{O}, 0.1 \% \mathrm{HCOOH}\right.$; $\mathrm{B}: \mathrm{CH}_{3} \mathrm{CN}, 0.1 \%$ $\mathrm{HCOOH}$; flow rate: $0.6 \mathrm{~mL} / \mathrm{min}$ ). A linear gradient of $15-75 \% \mathrm{~B}$ in 35 min was applied to separate all commercial available amino acid standards except of D- and D-allo-Ile. Separation of D- and D-allo-Ile was archived using chiral HPLC (CHIRALPAK IC, $1 \mathrm{~mL} / \mathrm{min}, 75 \% \mathrm{n}$-hexane, $25 \%$ isopropyl alcohol, $0.2 \% \mathrm{HCOOH}$ ). Prepared Marfey's adducts of synthesized $\beta$-hydroxyaspartic acids were analyzed using C18 RP-UHPLC-MS with a linear gradient of $5-20 \% \mathrm{~B}$ in $18 \mathrm{~min}$. For the determination of stereochemistry of $\beta$ hydroxyphenylalanines and $\beta$-hydroxyisoleucines, Cbz-protected intermediates were directly subjected to acidic hydrolysis $\left(6 \mathrm{M} \mathrm{HCl}, 2 \mathrm{~h}, 120^{\circ} \mathrm{C}\right)$ and subsequent treatment with Marfey's reagent on analytical scale as described before after drying. Marfey's adducts were analyzed using C18 RP-UHPLC-MS with 5-40\% B in 18 min.

Total hydrolysis of the chitinopeptins A-D was carried out by dissolving $250 \mu \mathrm{g}$ of each peptide in $6 \mathrm{M} \mathrm{DCl}$ in $\mathrm{D}_{2} \mathrm{O}$ and stirring for $7 \mathrm{~h}$ at $160{ }^{\circ} \mathrm{C}$. The samples were subsequently evaporated to dryness. Samples were dissolved in $100 \mu \mathrm{L} \mathrm{H}_{2} \mathrm{O}$, derivatized with l-FDVA and analyzed using the same parameters as described before.

Synthesis of $\beta$-hydroxyaspartic acids, $\beta$-hydroxyphenylalanines and $\beta$ hydroxyisoleucines: The syntheses of the $\beta$-hydroxyamino acids were achieved by modified literature known procedures. The enantiomeric excess of all synthesized amino acids were determined by chiral HPLC on selected intermediates. The synthesized compounds were fully characterized and/or compared with literature known references. For details of syntheses and characterization see Supporting Information.

\section{Optical rotation}

Specific rotation was determined on a digital polarimeter (P3000, A. Krüss Optronic GmbH, Germany). Standard wavelength was the sodium D-line with $589 \mathrm{~nm}$. Temperature, concentration $(\mathrm{g} / 100 \mathrm{~mL})$ and solvent are reported with the determined value.

\section{Minimal inhibitory concentration (MIC)}

The MIC was determined by broth microdilution method in 96 well plates with $100 \mu \mathrm{L}$ assay volume per well following EUCAST instructions $(113,114)$ for E. coli ATCC 35218, E. coli ATCC $25922 \Delta$ TolC, E. coli MG1655, $P$. aeruginosa ATCC 27853, $P$. aeruginosa PAO750, K. pneumoniae ATCC 13883, M. catarrhalis ATCC 25238, A. baumannii ATCC 19606, B. subtilis DSM 10, S. aureus ATCC 25923, and M. luteus DSM 20030. L. monocytogenes DSM 20600 (1 day of incubation at $37^{\circ} \mathrm{C}$ ) and M. smegmatis ATCC 607 ( 2 days of incubation at $37^{\circ} \mathrm{C}$ ) were grown in BHI broth supplemented with $1 \%(\mathrm{v} / \mathrm{v}$ ) Tween 80. C. albicans $\mathrm{FH} 2173$ was grown in MHIIB over 2 days of incubation at $37^{\circ} \mathrm{C}$ (preculture 2 days at $28^{\circ} \mathrm{C}$ ). Approximate inoculation cell density for all strains was $500.000 \mathrm{CFUs} / \mathrm{mL}$, except $1.000 .000 \mathrm{CFUs} / \mathrm{mL}$ were used for $M$. smegmatis and $C$. albicans. For all fungal screenings 100.000 spores $/ \mathrm{mL}$ were used and incubated at $37^{\circ} \mathrm{C}$ for A. flavus ATCC 9170 (MHIIB) and $25{ }^{\circ} \mathrm{C}$ for Z . tritici MUCL45407 (4 g/L yeast extract, $4 \mathrm{~g} / \mathrm{L}$ malt extract, $4 \mathrm{~g} / \mathrm{L}$ sucrose) and $F$. oxysporum ATCC 7601 (potato dextrose broth, Sigma). Growth inhibition was detected by optical density $(600 \mathrm{~nm})$ or for M. luteus, $L$. monocytogenes, $C$. albicans, $M$. smegmatis, and all fungi by quantification of free ATP using BacTiter-Glo after 24 to 48 hours (72 hours for Z tritici). All MIC assays were performed at least in triplicate. $\mathrm{FE}(\mathrm{III})$-citrate was added at molar ratios of 1:1 to samples in DMSO to test compounds in their iron bound conformation.

\section{References}

1. K. J. Fors berg, S. Patel, M. K. Gibson, C. L. Lauber, R. Knight, N. Fierer, G. Dantas, Bacterial phylogeny structures soil resistomes across habitats. Nature 509,612-616 (2014).

2. J. Nesme, P. Simonet, The soil resis tome: a critical review on antibiotic resistance origins, ecology and dissemination potential in telluric bacteria. Environmental microbiology 17,913-930 (2015).

3. K. J. Forsberg, A. Reyes, B. Wang, E. M. Selleck, M. O. A. Sommer, G. Dantas, The shared antibiotic resistome of soil bacteria and human pathogens. Science (New York, N.Y.) 337, 1107-1111 (2012). 
4. R. L. Finley, P. Collignon, D. G. J. Larsson, S. A. McEwen, X.-Z. Li, W. H. Gaze, R. Reid-Smith, M. Timinouni, D. W. Graham, E. Topp, The scourge of antibiotic resistance: the important role of the environment. Clinical infectious diseases : an official publication ofthe Infectious Diseases Society of America 57, 704-710 (2013).

5. UN Interagency Coordination Group (IACG) on Antimicrobial Resistance, No Time to Wait: Securing the future from drug-resistant infections (2019) (available at https://www.who.int/antimicrobial-resistance/interagencycoordination-group/final-report/en/).

6. E.-C. OERKE, Crop losses to pests. J.Agric. Sci. 144,31-43 (2006).

7. K. Kainz, M. A. Bauer, F. Madeo, D. Carmona-Gutierrez, Fungal infections in humans: the silent crisis . Microbial cell (Graz, Austria) 7, 143-145 (2020).

8. K. Lewis, Platforms for antibiotic discovery. Nat Rev Drug Discov 12, 371-387 (2013).

9. T. F. Schäberle, I. M. Hack, Overcoming the current deadlock in antibiotic research. Trends in Microbiology 22, 165-167 (2014).

10. D. J. Newman, G. M. Cragg, Natural Products as Sources of New Drugs over the Nearly Four Decades from 01/1981 to 09/2019. Journal of natural products 83,770-803 (2020).

11. T. Schneider, A. Müller, H. Miess, H. Gross, Cyclic lipopeptides as antibacterial agents - potent antibiotic activity mediated by intriguing mode of actions. International journal of medical microbiology: IJMM, 37-43 (2014).

12. C. Nord, J. Bjerketorp, J. J. Levenfors, S. Cao, A. A. Strömstedt, B. Guss, R. Larsson, D. Hughes, B. Öberg, A. Broberg, Isopedopeptins A-H: Cationic Cyclic Lipodepsipeptides from Pedobacter cryoconitis UP508 Targeting WHO Top-Priority Carbapenem-Resistant Bacteria. ACS chemical biology 15, 2937-2944 (2020).

13. M. Debono, M. Barnhart, C. B. Carrell, J. A. Hoffmann, J. L. Occolowitz, B. J. Abbott, D. S. Fukuda, R. L. Hamill, K. Biemann, W. C. Herlihy, A21978C, a complex of new acidic peptide antibiotics: isolation, chemistry, and mass spectral structure elucidation. The Journal of antibiotics 40,761-777 (1987).

14. R. D. Arbeit, D. Maki, F. P. Tally, E. Campanaro, B. I. Eisenstein, The safety and efficacy of daptomycin for the treatment of complicated skin and skin-structure infections. Clinical infectious diseases: an official publication of the Infectious Diseases Society of America 38, 1673-1681 (2004).

15. N. Vanittanakom, W. Loeffler, U. Koch, G. Jung, Fengycin--a novel antifungal lipopeptide antibiotic produced by Bacillus subtilis F-29-3. The Journal of antibiotics 39,888-901 (1986).

16. N. Geudens, J. C. Martins, Cyclic Lipodepsipeptides From Pseudomonas spp.- Biological Swiss-Army Knives. Front. Microbiol. 9, 1867 (2018).

17. W. Li, H. Rokni-Zadeh, M. de Vleeschouwer, M. G. K. Ghequire, D. Sinnaeve, G.-L. Xie, J. Rozenski, A. Madder, J. C. Martins, R. de Mot, The antimicrobial compound xantholysin defines a new group of Pseudomonas cyclic lipopeptides. PloS one 8, e62946 (2013).

18. F. E. Olorunleke, N. P. Kieu, M. Höfte, in Bacteria-Plant Interactions: Advanced Research and Future Trends (Caister Academic Press, 2015), pp. 167-198.

19. J. M. Raaijmakers, I. de Bruijn, O. Nybroe, M. Ongena, Natural functions of lipopeptides from Bacillus and Pseudomonas: more than surfactants and antibiotics. FEMS Microbiol Rev 34, 1037-1062 (2010).

20. P. Monciardini, M. Iorio, S. Maffioli, M. Sosio, S. Donadio, Discovering new bioactive molecules from microbial sources. Microbial Biotechnology 7, 209-220 (2014).

21. M. Adamek, M. Spohn, E. Stegmann, N. Ziemert, Mining Bacterial Genomes for Secondary Metabolite Gene Clusters. Methods in molecular biology(Clifton, N.J.) 1520,23-47 (2017).

22. N. Ziemert, M. Alanjary, T. Weber, The evolution of genome mining in microbes - a review. Nat. Prod. Rep. 33, 988-1005 (2016).

23. P. Cimermancic, M. H. Medema, J. Claesen, K. Kurita, L. C. Wieland Brown, K. Mavrommatis, A. Pati, P. A. Godfrey, M. Koehrsen, J. Clardy, B. W. Birren, E. Takano, A. Sali, R. G. Linington, M. A. Fischbach, Insights into secondary metabolism from a global analys is of prokaryotic biosynthetic gene clusters. Cell 158, 412-421 (2014).

24. K. L. Kurita, R. G. Linington, Connecting phenotype and chemotype: high-content discovery strategies for natural products research. Journal of natural products 78, 587-596 (2015).

25. M. H. Medema, M. A. Fischbach, Computational approaches to natural product discovery. Nat Chem Biol 11, 639-648 (2015).

26. J. C. Navarro-Muñoz, N. Selem-Mojica, M. W. Mullowney, S. A. Kautsar, J. H. Tryon, E. I. Parkinson, E. L. C. de Los Santos, M. Yeong, P. Cruz-Morales, S. Abubucker, A. Roeters, W. Lokhorst, A. Fernandez-Guerra, L. T. D. Cappelini, A. W. Goering, R. J. Thomson, W. W. Metcalf, N. L. Kelleher, F. Barona-Gomez, M. H. Medema, A computational framework to explore large-scale biosynthetic diversity. Nat Chem Biol 16, 60-68 (2020).

27. C. T. Walsh, M. A. Fis chbach, Natural products version 2.0: connecting genes to molecules. Journal of the American Chemical Society 132, 2469-2493 (2010).

28. J. M. Winter, S. Behnken, C. Hertweck, Genomics-inspired discovery of natural products. Current opinion in chemical biology 15, 22-31 (2011). 
29. J. J. J. van der Hooft, H. Mohimani, A. Bauermeister, P. C. Dorrestein, K. R. Duncan, M. H. Medema, Linking genomics and metabolomics to chart specialized metabolic diversity. Chemical Society reviews 49, 3297-3314 (2020).

30. J. R. Doroghazi, J. C. Albright, A. W. Goering, K.-S. Ju, R. R. Haines, K. A. Tchalukov, D. P. Labeda, N. L. Kelleher, W. W. Metcalf, A roadmap for natural product discovery based on large-scale genomics and metabolomics. Nat Chem Biol 10, 963-968 (2014).

31. M. A. Skinnider, C. W. Johnston, M. Gunabalasingam, N. J. Merwin, A. M. Kieliszek, R. J. MacLellan, H. Li, M. R. M. Ranieri, A. L. H. Webster, M. P. T. Cao, A. Pfeifle, N. Spencer, Q. H. To, D. P. Wallace, C. A. Dejong, N. A. Magarvey, Comprehensive prediction of secondary metabolite structure and biological activity from microbial genome sequences. Nat Commun 11, 6058 (2020).

32. T. Hoffmann, D. Krug, N. Bozkurt, S. Duddela, R. Jansen, R. Garcia, K. Gerth, H. Steinmetz, R. Müller, Correlating chemical diversity with taxonomic distance for discovery of natural products in my xobacteria. Nat Commun 9, 803 (2018).

33. B. Behsaz, E. Bode, A. Gurevich, Y.-N. Shi, F. Grundmann, D. Acharya, A. M. Caraballo-Rodríguez, A. Bouslimani, M. Panitchpakdi, A. Linck, C. Guan, J. Oh, P. C. Dorrestein, H. B. Bode, P. A. Pevzner, H. Mohimani, Integrating genomics and metabolomics for scalable non-ribosomal peptide discovery. Nat Commun 12, 3225 (2021).

34. V. L. Challinor, H. B. Bode, Bioactive natural products from novel microbial sources. Annals of the New York Academy of Sciences 1354, 82-97 (2015).

35. A. Crits-Christoph, S. Diamond, C. N. Butterfield, B. C. Thomas, J. F. Banfield, Novel soil bacteria possess diverse genes for secondary metabolite biosynthesis. Nature 558, 440-444 (2018).

36. L. L. Ling, T. Schneider, A. J. Peoples, A. L. Spoering, I. Engels, B. P. Conlon, A. Mueller, T. F. Schäb erle, D. E. Hughes, S. Epstein, M. Jones, L. Lazarides, V. A. Steadman, D. R. Cohen, C. R. Felix, K. A. Fetterman, W. P. Millett, A. G. Nitti, A. M. Zullo, C. Chen, K. Lewis, A new antibiotic kills pathogens without detectable resistance. Nature 517, 455-459 (2015).

37. Y. Imai, K. J. Meyer, A. Iinishi, Q. Favre-Godal, R. Green, S. Manuse, M. Caboni, M. Mori, S. Niles, M. Ghiglieri, C. Honrao, X. Ma, J. J. Guo, A. Makriyannis, L. Linares-Otoya, N. Böhringer, Z. G. Wuisan, H. Kaur, R. Wu, A. Mateus, A. Typas, M. M. Savitski, J. L. Espinoza, A. O'Rourke, K. E. Nelson, S. Hiller, N. Noinaj, T. F. Schäberle, A. D'Onofrio, K. Lewis, A new antibiotic selectively kills Gram-negative pathogens. Nature 576, 459-464 (2019).

38. F. Thomas, J.-H. Hehemann, E. Rebuffet, M. Czjzek, G. Michel, Environmental and gut bacteroidetes: the food connection. Front. Microbiol. 2,93 (2011).

39. R. Mendes, P. Garbeva, J. M. Raaijmakers, The rhizosphere microbiome: significance of plant beneficial, plant pathogenic, and human pathogenic microorganisms. FEMS Microbiol Rev 37, 634-663 (2013).

40. H. Steinmetz, K. Gerth, R. Jansen, N. Schläger, R. Dehn, S. Reinecke, A. Kirschning, R. Müller, Elansolid A, a unique macrolide antibiotic from Chitinophaga sancti isolated as two stable atropis omers. Angewandte Chemie International Edition 50, 532-536 (2011).

41. K. I. Mohr, C. Volz, R. Jansen, V. Wray, J. Hoffmann, S. Bernecker, J. Wink, K. Gerth, M. Stadler, R. Müller, Pinensins: the first antifungal lantibiotics. Angewandte Chemie International Edition 54, 11254-11258 (2015).

42. N. Katayama, Y. Nozaki, K. Okonogi, H. Ono, S. Harada, H. Okazaki, Formadicins, new monocyclic betalactam antibiotics of bacterial origin. I. Taxonomy, fermentation and biological activities. The Journal of antibiotics 38, 1117-1127 (1985).

43. N. Katayama, S. Fukusumi, Y. Funabashi, T. Iwahi, H. Ono, TAN-1057 A-D, new antibiotics with potent antibacterial activity against methicillin-resistant Staphylococcus aureus. Taxonomy, fermentation and biological activity. The Journal of antibiotics 46, 606-613 (1993).

44. J. Shoji, H. Hinoo, K. Matsumoto, T. Hattori, T. Yoshida, S. Matsuura, E. Kondo, Isolation and characterization of katanosins A and B. The Journal of antibiotics 41,713-718 (1988).

45. N. Oku, K. Adachi, S. Matsuda, H. Kasai, A. Takatsuki, Y. Shizuri, Ariakemicins A and B, novel polyketidepeptide antibiotics from a marine gliding bacterium of the genus Rapidithrix. Organic letters 10, 2481-2484 (2008).

46. C. Borsetto, G. C. A. Amos, U. N. da Rocha, A. L. Mitchell, R. D. Finn, R. F. Laidi, C. Vallin, D. A. Pearce, K. K. Newsham, E. M. H. Wellington, Microbial community drivers of PK/NRP gene diversity in selected global soils. Microbiome 7, 78 (2019).

47. R. L. Hahnke, J. P. Meier-Kolthoff, M. García-López, S. Mukherjee, M. Huntemann, N. N. Ivanova, T. Woyke, N. C. Kyrpides, H.-P. Klenk, M. Göker, Genome-Based Taxonomic Classification of Bacteroidetes. Front. Microbiol. 7, 2003 (2016).

48. R. L. Hahnke, J. P. Meier-Kolthoff, M. García-López, S. Mukherjee, M. Huntemann, N. N. Ivanova, T. Woyke, N. C. Kyrpides, H.-P. Klenk, M. Göker, Corrigendum: Genome-Based Taxonomic Classification of Bacteroidetes. Front. Microbiol. 9, 304 (2018).

49. K. Blin, S. Shaw, K. Steinke, R. Villebro, N. Ziemert, S. Y. Lee, M. H. Medema, T. Weber, antiSMASH 5.0: updates to the secondary metabolite genome mining pipeline. Nucleic acids research 47, W81-W87 (2019). 
50. K. C. Belknap, C. J. Park, B. M. Barth, C. P. Andam, Genome mining of biosynthetic and chemotherapeutic gene clusters in Streptomyces bacteria. Sci Rep 10, 2003 (2020).

51. A. Fajardo, J. L. Martínez, Antibiotics as signals that trigger specific bacterial responses. Current Opinion in Microbiology 11, 161-167 (2008).

52. S. C. Wenzel, R. Müller, The impact of genomics on the exploitation of the myxobacterial secondary metabolome. Nat. Prod. Rep. 26, 1385-1407 (2009).

53. M. H. Medema, P. Cimermancic, A. Sali, E. Takano, M. A. Fischbach, A systematic computational analysis of biosynthetic gene cluster evolution: les sons for engineering biosynthesis. PLoS computational biology $\mathbf{1 0}$, e1004016 (2014).

54. M. H. Medema, R. Kottmann, P. Yilmaz, M. Cummings, J. B. Biggins, K. Blin, I. de Bruijn, Y. H. Chooi, J. Claesen, R. C. Coates, P. Cruz-Morales, S. Duddela, S. Düsterhus, D. J. Edwards, D. P. Fewer, N. Garg, C. Geiger, J. P. Gomez-Escribano, A. Greule, M. Hadjithomas, A. S. Haines, E. J. N. Helfrich, M. L. Hillwig, K. Ishida, A. C. Jones, C. S. Jones, K. Jungmann, C. Kegler, H. U. Kim, P. Kötter, D. Krug, J. Masschelein, A. V. Melnik, S. M. Mantovani, E. A. Monroe, M. Moore, N. Moss, H.-W. Nützmann, G. Pan, A. Pati, D. Petras, F. J. Reen, F. Rosconi, Z. Rui, Z. Tian, N. J. Tobias, Y. Tsunematsu, P. Wiemann, E. Wyckoff, X. Yan, G. Yim, F. Yu, Y. Xie, B. Aigle, A. K. Apel, C. J. Balibar, E. P. Balskus, F. Barona-Gómez, A. Bechthold, H. B. Bode, R. Borris s, S. F. Brady, A. A. Brakhage, P. Caffrey, Y.-Q. Cheng, J. Clardy, R. J. Cox, R. de Mot, S. Donadio, M. S. Donia, W. A. van der Donk, P. C. Dorrestein, S. Doyle, A. J. M. Driessen, M. Ehling-Schulz, K.-D. Entian, M. A. Fischbach, L. Gerwick, W. H. Gerwick, H. Gross, B. Gust, C. Hertweck, M. Höfte, S. E. Jensen, J. Ju, L. Katz, L. Kaysser, J. L. Klassen, N. P. Keller, J. Kormanec, O. P. Kuipers, T. Kuzuyama, N. C. Kyrpides, H.-J. Kwon, S. Lautru, R. Lavigne, C. Y. Lee, B. Linquan, X. Liu, W. Liu, A. Luzhetskyy, T. Mahmud, Y. Mast, C. Méndez, M. Metsä-Ketelä, J. Micklefield, D. A. Mitchell, B. S. Moore, L. M. Moreira, R. Müller, B. A. Neilan, M. Nett, J. Nielsen, F. O'Gara, H. Oikawa, A. Osbourn, M. S. Osburne, B. Ostash, S. M. Payne, J.-L. Pernodet, M. Petricek, J. Piel, O. Ploux, J. M. Raaijmakers, J. A. Salas, E. K. Schmitt, B. Scott, R. F. Seipke, B. Shen, D. H. Sherman, K. Sivonen, M. J. Smanski, M. Sosio, E. Stegmann, R. D. Süssmu th, K. Tahlan, C. M. Thomas, Y. Tang, A. W. Truman, M. Viaud, J. D. Walton, C. T. Walsh, T. Weber, G. P. van Wezel, B. Wilkinson, J. M. Willey, W. Wohlleben, G. D. Wright, N. Ziemert, C. Zhang, S. B. Zotchev, R. Breitling, E. Takano, F. O. Glöckner, Minimum Information about a Biosynthetic Gene cluster. Nat Chem Biol 11, 625-631 (2015).

55. M. J. Fujita, K. Nakano, R. Sakai, Bisucaberin B, a linear hydroxamate class siderophore from the marine bacterium Tenacibaculummesophilum. Molecules (Basel, Switzerland) 18, 3917-3926 (2013).

56. L. Tao, H. Yao, H. Kasai, N. Misawa, Q. Cheng, A carotenoid synthes is gene cluster from Algoriphagus sp. KK10202C with a novel fusion-type lycopene beta-cyclase gene. Moleculargenetics and genomics : MGG, 7986 (2006).

57. M. J. McBride, G. Xie, E. C. Martens, A. Lapidus, B. Henrissat, R. G. Rhodes, E. Goltsman, W. Wang, J. Xu, D. W. Hunnicutt, A. M. Staroscik, T. R. Hoover, Y.-Q. Cheng, J. L. Stein, Novel features of the polysaccharidedigesting gliding bacterium Flavobacteriumjohnsoniae as revealed by genome sequence analysis. Applied and environmental microbiology 75, 6864-6875 (2009).

58. T. A. Schöner, S. W. Fuchs, C. Schönau, H. B. Bode, Initiation of the flexirubin biosynthesis in Chitinophaga pinensis. Microbial Biotechnology 7, 232-241 (2014).

59. P. D. Singh, J. H. Johnson, P. C. Ward, J. S. Wells, W. H. Trejo, R. B. Sykes, SQ 28,332, a new monobactam produced by a Flexibacter sp. Taxonomy, fermentation, isolation, structure determination and biological properties. The Journal of antibiotics 36, 1245-1251 (1983).

60. Webster A. L. H., C. Walker, Li H., Skinnider M., Magarvey N. A., [Flexibacter] sp. ATCC 35208 monobactam biosynthesis gene cluster, Accession no. KY502195.1 (GenBank) (available at https://www.ncbi.nlm.nih.gov/nuccore/KY502195.1).

61. R. Dehn, Y. Katsuyama, A. Weber, K. Gerth, R. Jansen, H. Steinmetz, G. Höfle, R. Müller, A. Kirschning, Molecular basis of elansolid biosynthesis: evidence for an unprecedented quinone methide initiated intramolecular Diels-Alder cycloaddition/macrolactonization. Angewandte Chemie International Edition 50, 3882-3887 (2011).

62. K. Gerth, R. Jansen, K. I. Mohr, R. Müller, C. Volz, V. Wray, NOVELLANTIBIOTICS (2016) WO2016045783A1.

63. R. Teta, M. Gurgui, E. J. N. Helfrich, S. Künne, A. Schneider, G. van Echten-Deckert, A. Mangoni, J. Piel, Genome mining reveals trans-AT polyketide synthase directed antibiotic biosynthesis in the bacterial phylum bacteroidetes. Chembiochem : a European journal of chemical biology 11, 2506-2512 (2010).

64. K. Gerth, H. Steinmetz, G. Höfle, ELANSOLIDS, NOVEL NATURAL METABOLITES OF FLEXIBACTER AND ANTIBIOTICALLY ACTIVE DERIVATIVES THEREOF (19.02.2009) US20110034528.

65. H. B. Bode, B. Bethe, R. Höfs, A. Zeeck, Big Effects from Small Changes: Possible Ways to Explore Nature's Chemical Diversity. Chembiochem: a European journal of chemical biology 3, 619 (2002).

66. M. Urem, M. A. Świątek-Połatyńska, S. Rigali, G. P. van Wezel, Intertwining nutrient-sensory networks and the control of antibiotic production in Streptomyces. Molecular Microbiology 102, 183-195 (2016). 
67. W. Wohlleben, A. Bera, Y. Mast, E. Stegmann, in Biology and Biotechnology ofActinobacteria, J. Wink, F. Mohammadipanah, J. Hamedi, Eds. (Springer International Publishing, Cham, 2017), pp. 181-232.

68. R. Stolt, R. J. O. Torgrip, J. Lindberg, L. Csenki, J. Kolmert, I. Schuppe-Koistinen, S. P. Jacobsson, Secondorder peak detection for multicomponent high-resolution LC/MS data. Analytical chemistry 78, 975-983 (2006).

69. H. Laatsch, AntiBase: The Natural Compound Identifier (Wiley-VCH, Weinheim, Germany, ed. 5, 2017).

70. B. Somanadhan, S. R. Kotturi, C. Yan Leong, R. P. Glover, Y. Huang, H. Flotow, A. D. Buss, M. J. Lear, M. S. Butler, Isolation and synthesis of falcitidin, a novel myxo bacterial-derived acyltetrapeptide with activity against the malaria target falcipain-2. The Journal of antibiotics 66, 259-264 (2013).

71. R. Bhushan, H. Brückner, Marfey's reagent for chiral amino acid analysis: a review. Amino acids 27, 231-247 (2004).

72. A. Breuning, R. Vicik, T. Schirmeister, An improved synthesis of aziridine-2,3-dicarboxylates via azido alcohols - epimerization studies. Tetrahedron: Asymmetry 14, 3301-3312 (2003).

73. M. A. Blaskovich, G. A. Lajoie, Synthesis of a chiral serine aldehy de equivalent and its conversion to chiral .alpha.-amino acid derivatives. J. Am. Chem. Soc. 115, 5021-5030 (1993).

74. M. A. Blaskovich, G. Evindar, N. G. W. Rose, S. Wilkinson, Y. Luo, G. A. Lajoie, Stereoselective Synthesis of Threo and Erythro $\beta$-Hydroxy and $\beta$-Disubstituted- $\beta$-Hydroxy $\alpha$-Amino Acids. J. Org. Chem. 63, 3631-3646 (1998).

75. D. B. Hansen, M. M. Joullié, A stereoselective synthesis of (2S,3R)- $\beta$-methoxyphenylalanine: a component of cyclomarin A. Tetrahedron: Asymmetry 16, 3963-3969 (2005).

76. R. C. Hider, X. Kong, Chemistry and biology of siderophores. Nat. Prod. Rep. 27, 637-657 (2010).

77. M. Marner, M. A. Patras, M. Kurz, F. Zubeil, F. Förster, S. Schuler, A. Bauer, P. Hammann, A. Vilcinskas, T. F. Schäberle, J. Glaeser, Molecular Networking-Guided Discovery and Characterization of Stechlisins, a Group of Cyclic Lipopeptides from a Pseudomonas sp. Journal of natural products 83, 2607-2617 (2020).

78. T. M. Makris, M. Chakrabarti, E. Münck, J. D. Lipscomb, A family of diiron monooxyg enases catalyzing amino acid beta-hydroxylation in antibiotic biosynthesis. Proceedings of the National Academy of Sciences 107, 1539115396 (2010).

79. J. Cheung, F. C. Beasley, S. Liu, G. A. Lajoie, D. E. Heinrichs, Molecular characterization of staphylo ferrin B biosynthesis in Staphylococcus aureus. Molecular Microbiology 74, 594-608 (2009).

80. F. C. Beasley, J. Cheung, D. E. Heinrichs, Mutation of L-2,3-diaminopropionic acid synthase genes blocks staphyloferrin B synthesis in Staphylococcus aureus. BMC Microbiol 11, 199 (2011).

81. J. H. Carter, R. H. Du Bus, J. R. Dyer, J. C. Floyd, K. C. Rice, P. D. Shaw, Biosynthesis of viomycin. I. Origin of alpha, beta-diaminopropionic acid and serine. Biochemistry 13, 1221-1227 (1974).

82. M. Wang, S. J. Gould, Biosynthesis of capreomycin. 2. Incorporation of L-serine, L-alanine, and L-2,3diaminopropionic acid. J. Org. Chem. 58, 5176-5180 (1993).

83. E. A. Felnagle, M. R. Rondon, A. D. Berti, H. A. Crosby, M. G. Thomas, Identification of the biosynthetic gene cluster and an additional gene for resistance to the antituberculosis drug capreomycin. Applied and environmental microbiology 73, 4162-4170 (2007).

84. C. Zhao, C. Song, Y. Luo, Z. Yu, M. Sun, L-2,3-diaminopropionate: one of the building blocks for the biosynthesis ofZwittermicin A in Bacillus thuringiensis subsp. kurstaki strain YBT-1520. FEBS letters 582, 3125-3131 (2008).

85. M. Spohn, W. Wohlleben, E. Stegmann, Elucidation of the zinc-dependent regulation in Amycolatopsis japonicum enabled the identification of the ethylenediamine-disuccinate (S,S-EDDS) genes. Environmental microbiology 18, 1249-1263 (2016).

86. M. Spohn, S. Edenhart, M. Alanjary, N. Ziemert, D. Wibberg, J. Kalinowski, T. H. J. Niedermeyer, E. Stegmann, W. Wohlleben, Identification of a novel aminopolycarboxylic acid siderophore gene cluster encoding the biosynthesis of ethylenediaminesuccinic acid hydroxyarginine (EDHA). Metallomics 10, 722-734 (2018).

87. T. Stachelhaus, H. D. Mootz, M. A. Marahiel, The specificity-conferring code of adenylation domains in nonribosomal peptide synthetases. Chemistry \& biology 6, 493-505 (1999).

88. A. W. Goering, R. A. McClure, J. R. Doroghazi, J. C. Albright, N. A. Haverland, Y. Zhang, K.-S. Ju, R. J. Thomson, W. W. Metcalf, N. L. Kelleher, Metabologenomics: Correlation of Microbial Gene Clusters with Metabolites Drives Discovery of a Nonribosomal Peptide with an Unusual Amino Acid Monomer. ACS central science 2, 99-108 (2016).

89. J. A. van Santen, S. A. Kautsar, M. H. Medema, R. G. Linington, Microbial natural product databases:moving forward in the multi-omics era. Nat. Prod. Rep. 38, 264-278 (2021).

90. R. H. Baltz, Gifted microbes for genome mining and natural product discovery. Journal of industrial microbiology \& biotechnology 44, 573-588 (2017).

91. M. Adamek, M. Alanjary, H. Sales-Ortells, M. Goodfellow, A. T. Bull, A. Winkler, D. Wibberg, J. Kalinowski, N. Ziemert, Comparative genomics reveals phylogenetic distribution patterns of secondary metabolites in Amycolatopsis species. BMC genomics 19, 426 (2018).

92. Buckingham J., Dictionary of natural products 29.1 Chemical Search. (available at http://dnp.chemnetbase.com/faces/chemical/ChemicalSearch.xhtml). 
93. T. Janek, L. R. Rodrigues, E. J. Gudiña, Ż. Czyżnikowska, Structure and mode of action of cyclic lipopeptide pseudofactin II with divalent metal ions. Colloids and surfaces. B, Biointerfaces 146, 498-506 (2016).

94. E. Ksiezopolska, T. Gabaldón, Evolutionary Emergence of Drug Resistance in Candida Opportunistic Pathogens. Genes 9 (2018).

95. F. C. Odds, A. J.P. Brown, N. A.R. Gow, Antifungal agents:mechanisms of action. Trends in Microbiology 11, 272-279 (2003).

96. A. Mhammedi, F. Peypoux, F. Besson, G. Michel, Bacillomycin F, a new antibiotic of iturin group: isolation and characterization. The Journal of antibiotics 35, 306-311 (1982).

97. K. R. Meena, S. S. Kanwar, Lipopeptides as the antifungal and antibacterial agents:applications in food safety and therapeutics. BioMed research international 2015, 473050 (2015).

98. R. Maget-Dana, M. Ptak, F. Peypoux, G. Michel, Pore-forming properties of iturin A, a lipopeptide antibiotic. Biochimica et Biophysica Acta (BBA) - Biomembranes 815, 405-409 (1985).

99. R. Maget-Dana, I. Harnois, M. Ptak, Interactions of the lipopeptide antifungal iturin A with lipids in mixed monolayers. Biochimica et Biophysica Acta (BBA) - Biomembranes 981, 309-314 (1989).

100. M. Deleu, M. Paquot, T. Nylander, Fengycin interaction with lipid monolayers at the air-aqueous interfaceimplications for the effect of fengycin on biological membranes. Journal of colloid and interface science $\mathbf{2 8 3}$, 358-365 (2005).

101. M. Deleu, M. Paquot, T. Nylander, Effect of fengycin, a lipopeptide produced by Bacillus subtilis, on model biomembranes. Biophysical journal 94, 2667-2679 (2008).

102. NCBI Resource Coordinators, Database resources of the National Center for Biotechnology Information. Nucleic Acids Res 46, D8-D13 (2018).

103. V. M. Markowitz, I.-M. A. Chen, K. Palaniappan, K. Chu, E. Szeto, Y. Grechkin, A. Ratner, B. Jacob, J. Huang, P. Williams, M. Huntemann, I. Anderson, K. Mavromatis, N. N. Ivanova, N. C. Kyrpides, IMG: the Integrated Microbial Genomes database and comparative analysis system. Nucleic Acids Res 40, D115-22 (2012).

104. K. Katoh, K. Misawa, K.-i. Kuma, T. Miyata, MAFFT: a novel method for rapid multiple sequence alignment based on fast Fourier transform. Nucleic Acids Res 30, 3059-3066 (2002).

105. A. Stamatakis, RAxML version 8: a tool for phylogenetic analysis and post-analysis of large phylogenies. Bioinformatics 30, 1312-1313 (2014).

106. S. TAVARE, Some probabilistic and statistical problems in the analysis of DNA sequences. American Mathematical Society: Lectures on Mathematics in the Life Sciences 17, 57-86 (1986).

107. I. Letunic, P. Bork, Interactive Tree Of Life (iTOL) v4: recent updates and new developments. Nucleic Acids Res 47, W256-W259 (2019).

108. J. D. Thompson, D. G. Higgins, T. J. Gibson, CLUSTAL W: improving the sensitivity of progressive multiple sequence alignment through sequence weighting, position-specific gap penalties and weight matrix choice. Nucleic Acids Res 22, 4673-4680 (1994).

109. S. Kumar, G. Stecher, K. Tamura, MEGA7: Molecular Evolutionary Genetics Analysis Version 7.0 for Bigger Datasets. Molecularbiology and evolution 33, 1870-1874 (2016).

110. P. Shannon, A. Markiel, O. Ozier, N. S. Baliga, J. T. Wang, D. Ramage, N. Amin, B. Schwikowski, T. Ideker, Cytoscape: a software environment for integrated models of biomolecular interaction networks. Genome research 13, 2498-2504 (2003).

111. D. J. Reasoner, E. E. Geldreich, A new medium for the enumeration and subculture of bacteria from potable water. Applied and environmental microbiology 49, 1-7 (1985).

112. F. Widdel, G.-W. Kohring, F. Mayer, Studies on dissimilatory sulfate-reducing bacteria that decompose fatty acids. Arch. Microbiol. 134, 286-294 (1983).

113. EUCAST, Determination of minimum inhibitory concentrations (MICs) of antibacterial agents by broth dilution. Clinical Microbiology and Infection 9, ix-xv (2003).

114. EUCAST definitive document EDef 7.1: method for the determination of broth dilution MICs of antifungal agents for fermentative yeasts. Clinical microbiology and infection : the official publication of the European Society of Clinical Microbiology and Infectious Diseases 14, 398-405 (2008).

\section{Acknowledgments}

The authors intend to show a deep sense of gratitude to Sanja Mihajlovic as caretaker of the biobank. The authors also thank Mona-Katharina Bill, Sandra Semmler, Sören M. M. Schuler, Frank Förster, Christine Wehr, Jennifer Kuhn, Nadine Zucchetto, Sina Serife Abdo, Regina Zweigert, Mona Abdullahi, and Kirsten-Susann Bommersheim for their support and valuable discussions as well as Heiko Heese and Joachim Kluge for HPLC purifications of synthetic intermediates and Karin Rahn-Hotze for ee-determininations. We 
thank Stefan Bernhardt (Institute for Organic Chemistry, Justus-Liebig-University Giessen) for chiral HPLC analysis.

Funding: This work was financially supported by the Hessen State Ministry of Higher Education, Research and the Arts (HMWK) via the state initiative for the development of scientific and economic excellence for the LOEWE Center for Insect Biotechnology and Bioresources. Sanofi-Aventis Deutschland $\mathrm{GmbH}$ and Evotec International $\mathrm{GmbH}$ funded this work in the framework of the Sanofi-Fraunhofer Natural Products Center and its follow up, the Fraunhofer-Evotec Natural Products Center of Excellence.

Author contributions: $\mathrm{SBr}$ and MSS conceived and designed the experiments. SBr, MSS, MAP, BL, MM, CH, ABi, SBe, YK, CP, MK performed the experiments. SBr, MSS, MAP, $\mathrm{CP}, \mathrm{YK}, \mathrm{ABa}, \mathrm{LT}, \mathrm{MK}$, TFS analyzed the data. ABa, LT, JG, PEH initiated the project idea. JG, MSS, TFS supervised the project. AV and PEH initiated the public-private partnership between Fraunhofer and Sanofi (later Evotec). SBr, MSS, TFS drafted the first manuscript. $\mathrm{SBr}$, MSS and TFS revised the manuscript. All authors accepted the final version of the manuscript.

Competing interests: The authors declare no conflict of interest. The authors declare no competing financial interest.

Data and materials availability: All data are available in the main text or the supplementary materials. 
Figures
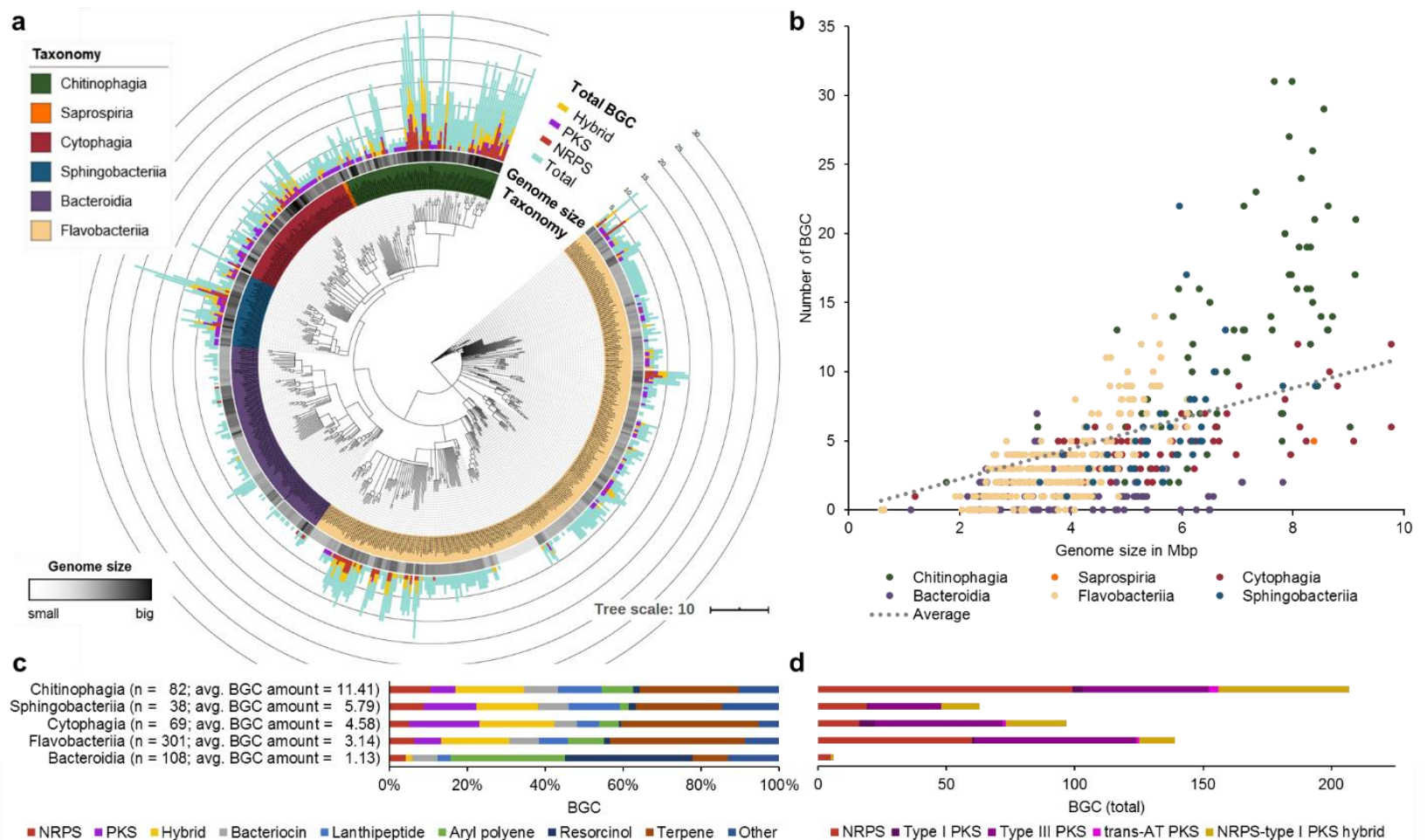

Fig. 1. Bioinformatics analysis of 600 genomes of the phylum Bacteroidetes points towards high biosynthetic genetic potential of the Chitinophagia class. (A) Consensus tree based on maximum-likelihood method (RAxML model v8(105), GTR GAMMA with 1000 bootstraps) of $60016 \mathrm{~S}$ rRNA gene sequences color coded on class level. For each strain the genome size and biosynthetic gene cluster amount and types are depicted. Tree is annotated using iTOL v4(107). (B) Correlation of the total gene cluster amount with the genome size of each stain. (C) Analys is of the BGC types in the individual classes. BGC types: NRPS, nonribosomal peptide; PKS, polyketide; hybrid, cluster containing more than one BGC type; and other, remaining BGC types not separately listed. (D) Detailed look onto the most essential BGC types responsible for the production of bioactive NPs. Partial BGCs on contigs $<10 \mathrm{~kb}$ of WGS projects are not included in all graphs. 


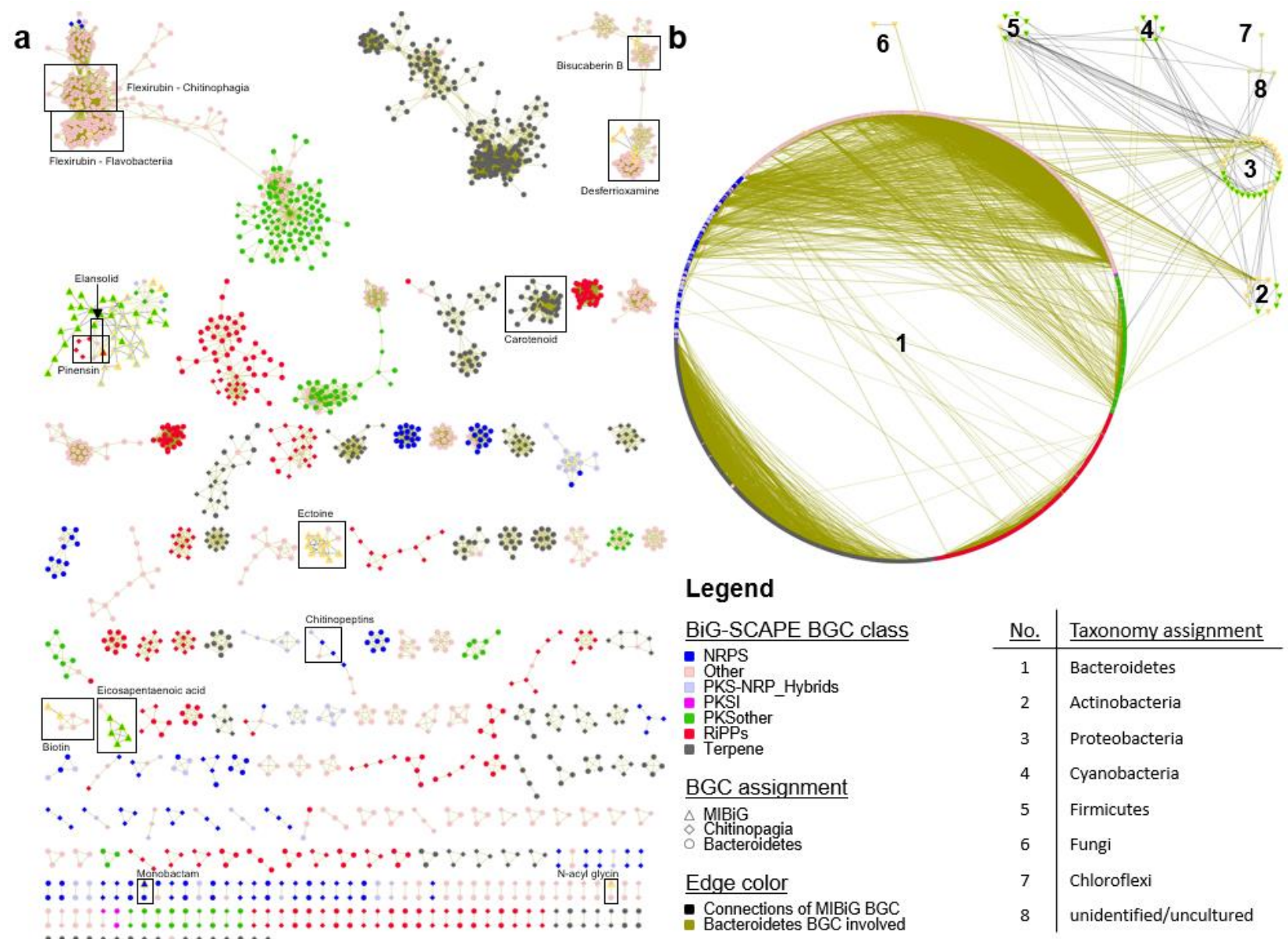

Fig. 2. BiG-SCAPE(26) analysis of the phylum Bacteroidetes highlights a giant uncovered genetic potential. (A) A global network of all depicted gene cluster families with a cutoff of 0.6. Known ones are marked, named and are identified by known biosynthetic gene cluster (BGC) deposited at MIBiG (all known and deposited Bacteroidetes BGCs are found). (B) Same BGCs sorted by taxonomy. BiG-SCAPE BGC classes: NRPS, nonribosomal peptide; PKS, polyketide; and RiPPs, ribosomally synthes ised and posttranslational modified peptides. Singeltons (unique BGCs without any connection) are not shown. Visualization and manipulation by Cytoscape v3.4.0(110). 
bioRxiv preprint doi: https://doi.org/10.1101/2021.07.30.454449; this version posted July 30, 2021. The copyright holder for this preprint (which was not certified by peer review) is the author/funder, who has granted bioRxiv a license to display the preprint in perpetuity. It is made available under aCC-BY-NC-ND 4.0 International license.

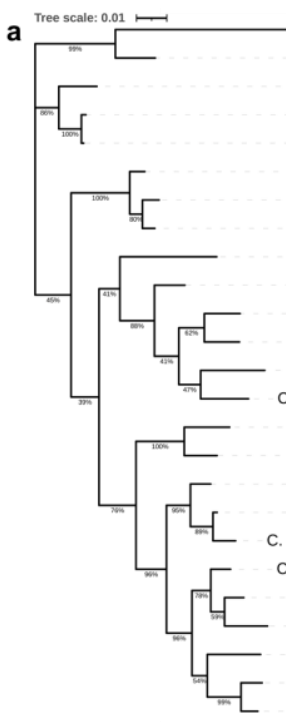

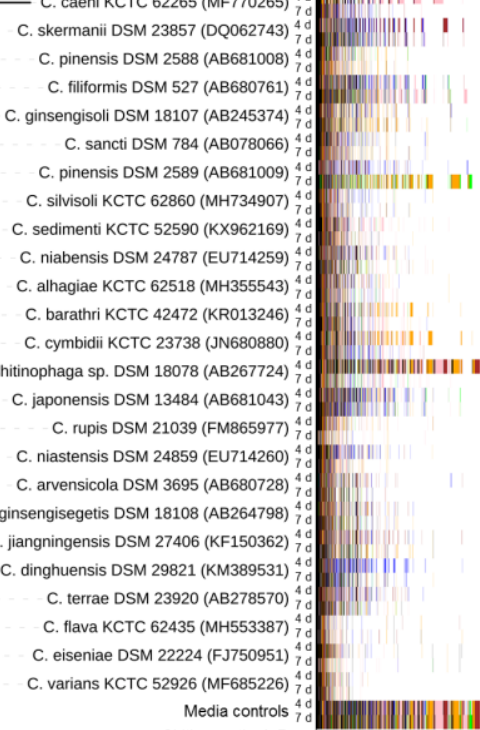

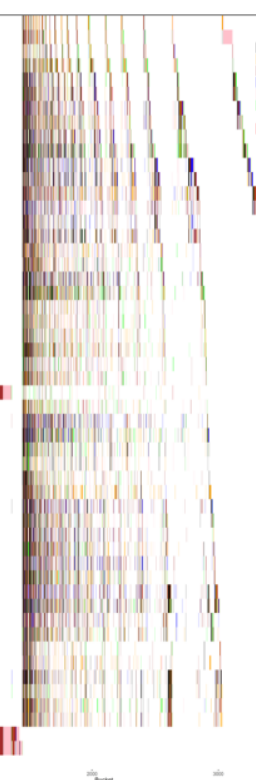

b

Unique "metabolite" buckets per strain
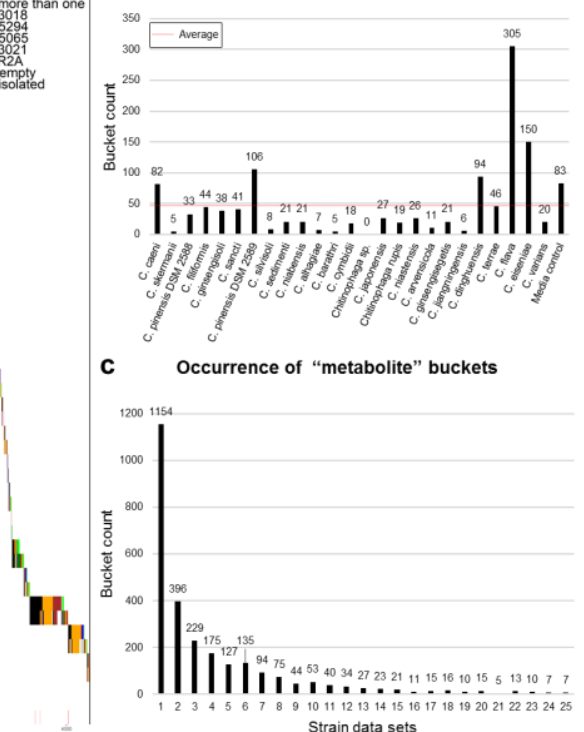

Occurrence of "metabolite" buckets

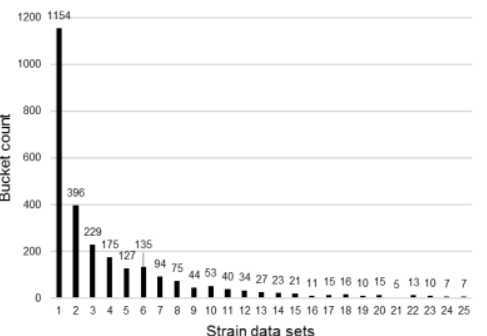

Fig. 3. Taxonomical arrayed chemotype-barcoding matrix reveals an uncharted chemical space within the genus Chitinophaga. (A) The tree is based on a Clustal W alignment(108) of available 16S rRNA gene sequences of 25 Chitinophaga strains available for cultivation. The tree was calculated using MEGA v7.0.26 with the maximum-likelihood method and GTR-Gamma model(109). Percentage on the tree branches indicate values of 1,000 bootstrap replicates with a bootstrap support of more than $50 \%$. The tree is drawn to scale, with branch lengths measured in the number of substitutions per site. The bucketing process is depicted as a chemotype-barcode matrix and color coded by the condition each individual bucket was present. (B) Bar blot of the unique "metabolite" buckets of each strain. (C) Bar blot depicting the occurrence of "metabolite" buckets in the datasets. 


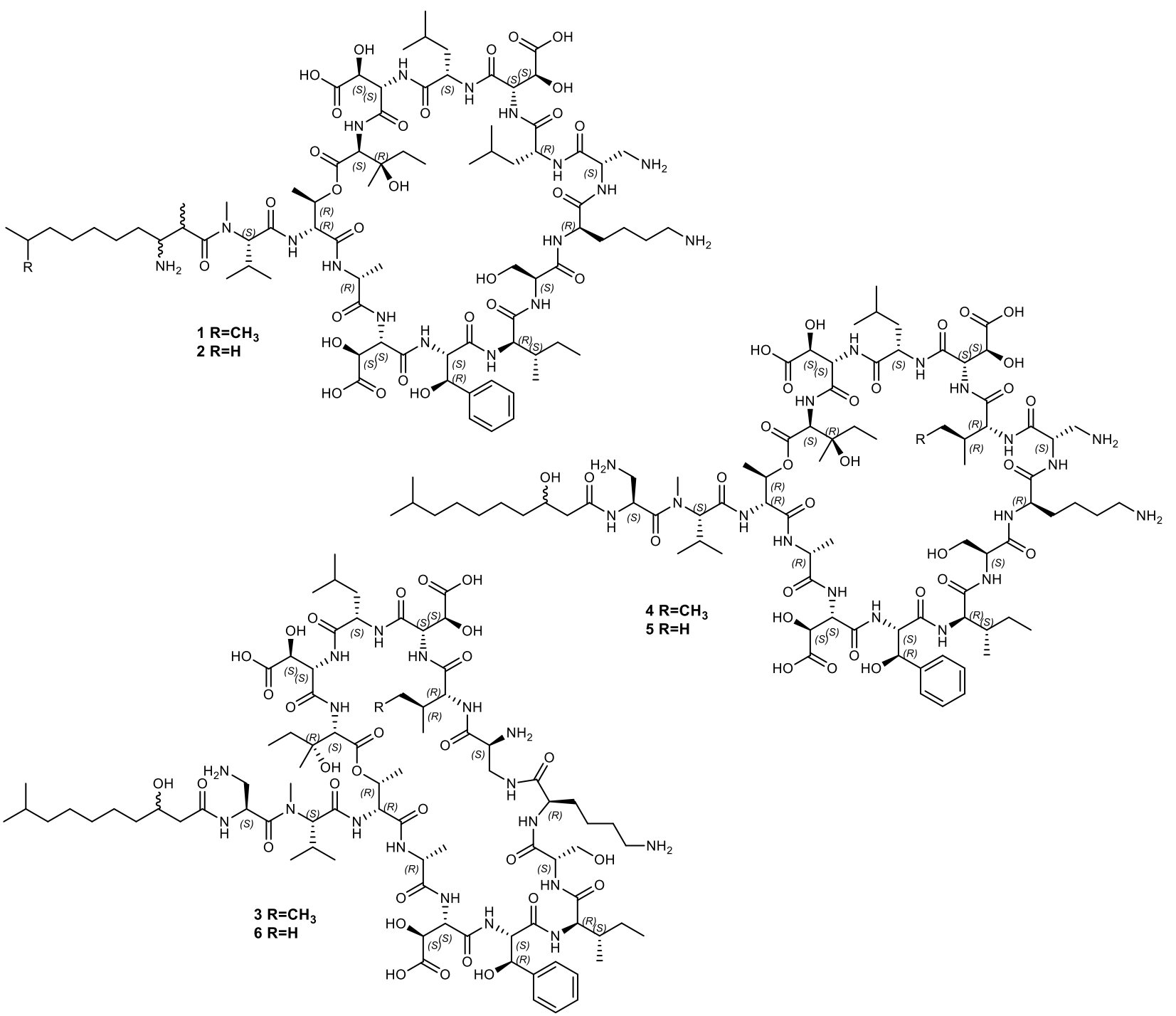

Fig. 4. Chemical structures of chitinopeptin A-D (1-6) produced by C. eiseniae DSM $22224(1,2)$ and C. flava KCTC 62435 (3-6). 


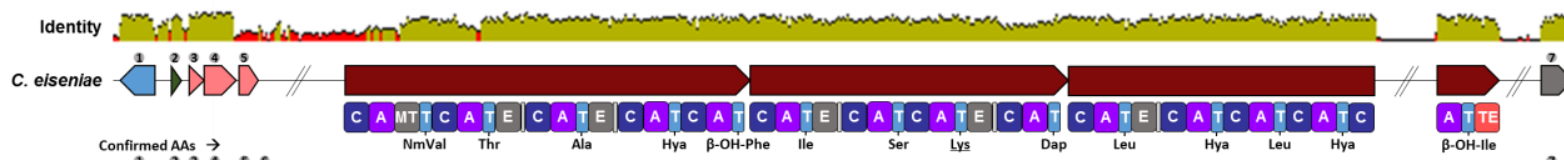

\section{Supplementary Materials}

(1) General supporting material

(2) Synthesis of beta-hydroxy amino acid supporting material

(3) Supplementary Table 8 